\title{
Nitrogen-doped CVD diamond: Nitrogen concentration, color and internal stress
}

\author{
A.M. Zaitsev ${ }^{\mathrm{a}, \mathrm{b}, *}$, N.M. Kazuchits ${ }^{\mathrm{c}}$, V.N. Kazuchits ${ }^{\mathrm{c}}$, K.S. Moe ${ }^{\mathrm{b}}$, M.S. Rusetsky ${ }^{\mathrm{c}}$, O.V. Korolik ${ }^{\mathrm{c}}$, \\ Kouki Kitajima ${ }^{\mathrm{d}}$, J.E. Butler ${ }^{\mathrm{e}}$, W. Wang ${ }^{\mathrm{b}}$ \\ ${ }^{\text {a }}$ The College of Staten Island/CUNY, 2800 Victory Blvd., Staten Island, NY 10312, USA \\ ${ }^{\mathrm{b}}$ Gemological Institute of America, $50 \mathrm{~W}$ 47th St \#800, New York, NY 10036, USA \\ ${ }^{\mathrm{c}}$ Belarusian State University, Nezavisimosti Ave. 4, 220030 Minsk, Belarus \\ ${ }^{\mathrm{d}}$ Department of Geoscience, University of Wisconsin Madison, 1215 W. Dayton St., WI 53706, USA

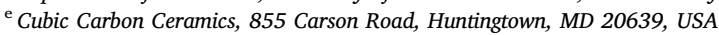

A R T I C L E I N F O

\section{Keywords:}

CVD diamond

Nitrogen doping

Birefringence

SIMS

Brown gray color

Stress

Carbon nanoclusters

\begin{abstract}
A B S T R A C T
Single crystal CVD diamond has been grown on (100)-oriented CVD diamond seed in six layers to a total thickness of $4.3 \mathrm{~mm}$, each layer being grown in gas with increasing concentration of nitrogen. The nitrogen doping efficiency, distribution of color and internal stress have been studied by SIMS, optical absorption, Raman spectroscopy and birefringence imaging. It is shown that nitrogen doping is very non-uniform. This non-uniformity is explained by the terraced growth of CVD diamond. The color of the nitrogen-doped diamond is grayish-brown with color intensity gradually increasing with nitrogen concentration. The absorption spectra are analyzed in terms of two continua representing brown and gray color components. The brown absorption continuum exponentially rises towards short wavelength. Its intensity correlates with the concentration of nitrogen C-defects. Small vacancy clusters are discussed as the defects responsible for the brown absorption continuum. The gray absorption continuum has weak and almost linear spectral dependence through the near infrared and visible spectral range. It is ascribed to carbon nanoclusters which may form in plasma and get trapped into growing diamond. It is suggested that Mie light scattering on the carbon nanoclusters substantially contributes to the gray absorption continuum and determines its weak spectral dependence. A Raman line at a wavenumber of $1550 \mathrm{~cm}^{-1}$ is described as a characteristic feature of the carbon nanoclusters. The striation pattern of brown/gray color follows the pattern of anomalous birefringence suggesting that the vacancy clusters and carbon inclusions are the main cause of internal stress in CVD diamond. A conclusion is made that high perfection of seed surface at microscale is not a required condition for growth of low-stress, low-inclusion single crystal CVD diamond. Crystallographic order at macroscale is more important requirement for the seed surface.
\end{abstract}

\section{Introduction}

Nitrogen is the major impurity in diamond. Nitrogen influences all physical properties of diamond and especially its optical, electronic, thermal and gemological properties. Nitrogen in diamond has been rigorously studied for decades and now it is one of the most studied impurities in solids. Although nitrogen doping and behavior of nitrogen have been thoroughly studied in natural and high pressure high temperature (HPHT) grown synthetic diamonds, there is still no clear understanding of the behavior of nitrogen in chemical vapor deposition (CVD) grown diamonds. As CVD diamond is becoming increasingly the material of choice in many areas of research and industry, the lack of the detailed information on the processes of nitrogen doping and transformation of nitrogen-related defects in CVD diamond is becoming an issue.

Natural and HPHT synthetic diamonds grow at high pressure under conditions where diamond is the thermodynamically stable form of crystalline carbon. In contrast, the growth of CVD diamond at low pressure, where diamond is not thermodynamically stable, is a highly non-equilibrium process [1]. The chemistry of the growth media for CVD diamond is also different from that in which natural and HPHT synthetic diamonds grow. Since the stability of nitrogen-containing defects and the kinetics of their formation strongly depend on pressure, chemical environment, and time at temperatures relevant to bulk atomic diffusion, the behavior of nitrogen in CVD diamond is expected to differ from that observed in natural and HPHT-grown synthetic

\footnotetext{
* Corresponding author at: The College of Staten Island/CUNY, 2800 Victory Blvd., Staten Island, NY 10312, USA.

E-mail address: alexander.zaitsev@csi.cuny.edu (A.M. Zaitsev).
} 


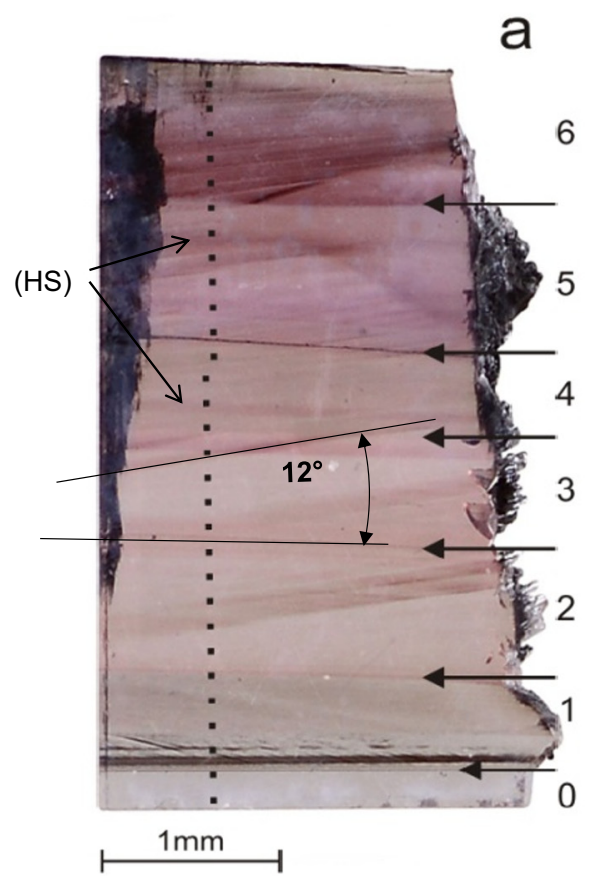

5

4

3

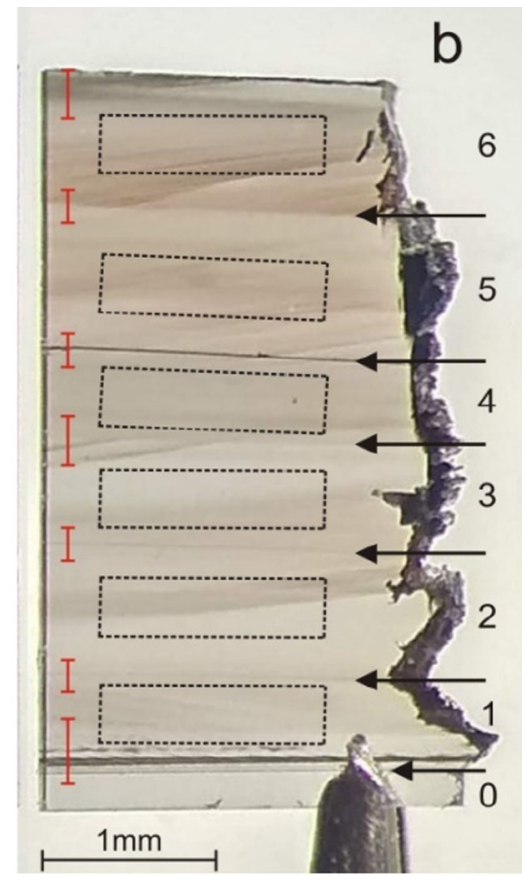

Fig. 1. (a) Image of plate \#5293-4 after irradiation and annealing. The dots show the places of SIMS measurements. Angle $12^{\circ}$ shows preferential orientation of colored striations with respect to growth interfaces. The horizontal striations running parallel to the interlayer boundaries are labeled with (HS). (b) Image of plate \#5293-5 used for absorption and Raman measurements. Positions of interlayer boundaries are shown with arrows. The dashed line rectangles show the areas selected for absorption measurements. Red vertical lines show the ranges of scans where detailed Raman measurements were performed. The growth layers are labeled with numbers 1 to 6 . The substrate is labeled with 0 . (For interpretation of the references to color in this figure legend, the reader is referred to the web version of this article.) diamonds.

The most common well-characterized nitrogen-related defects in natural diamonds are the C-defect (single nitrogen atom in a lattice site), the A-defect (two adjacent nitrogen atoms in lattice sites), the Bdefect (four substitutional nitrogen atoms surrounding a vacant lattice site), the N3-defect (three nitrogen atoms around a vacancy), the H3defect (two substitutional nitrogen atoms bound to vacancy) and the NV-defect (close pair of substitutional nitrogen atom and vacancy) (for quick reference see [2]). In HPHT metal catalyst grown diamonds, the common form of nitrogen is the C-defect in neutral charge state. In CVD-grown diamond, nitrogen is generally detected as C-defects, NVdefects and NVH-defects (a hydrogen atom attached to NV-defect). Some of these defects are also known to exist in several charge states (e.g. [3-5]).

Some peculiarities of behavior of nitrogen in CVD diamond are the low doping efficiency during growth [6-8], the low aggregation rate at high temperature (negligible formation of A- and B-defects), and the formation of many specific defects never observed in HPHT-grown synthetic and natural diamonds (e.g. $[9,10]$ ). In order to get a better insight in the behavior of nitrogen in CVD diamond we studied nitrogen-doped CVD diamond and, in this communication, we present the results on the efficiency of doping, internal stress and formation of major defects determining color of CVD diamond grown in the presence of nitrogen.

\section{Experimental}

The studies were performed on a CVD diamond grown in the GIA CVD Diamond Lab using the PLASSYS 150 microwave plasma assisted CVD tool (microwave frequency $2.45 \mathrm{GHz}$ ). The diamond block \#5293 was grown on a CVD substrate of size $5 \times 5 \times 0.25 \mathrm{~mm}^{3}$ to a total thickness of $4.3 \mathrm{~mm}$. This substrate was purchased from third parties and no information about its growth was available. Preliminary spectroscopic characterization revealed that the substrate was of a high structural perfection, had concentration of optically active nitrogen < $1 \mathrm{ppm}$, and had been annealed at high temperature. Most probably it was HPHT annealing performed with the aim to remove brownish color, which is typical for commercial as-grown CVD diamond.

The CVD growth was performed in plasma of composition $95.8 \%$ of
$\mathrm{H}_{2}$ and $4.2 \%$ of $\mathrm{CH}_{4}$ at a pressure of 360 mbar with gas flow rate $150 \mathrm{sccm}$ and microwave power about $3.8 \mathrm{~kW}$. The growth was performed in 6 runs forming 6 distinctive growth layers. The growth procedure was one and the same for each layer: ultrasonic cleaning of sample in acetone, pumping out the growth chamber of the reactor to a vacuum $5 \times 10^{-4} \mathrm{mbar}$, cleaning the sample surface in plasma $(90 \%$ $\mathrm{H}_{2}, 10 \% \mathrm{O}_{2}$ ) for about 20 min with gradual pressure rising from 10 to 360 mbar, power rising from 1.5 to $3.8 \mathrm{~kW}$ and temperature rising from room temperature to $950{ }^{\circ} \mathrm{C}$. During growth, the temperature of the diamond was kept at $950{ }^{\circ} \mathrm{C}$. As the diamond grew and became thicker, the distance between its growth surface and the plasma ball was adjusted by vertical movements of the sample holder.

The concentration of impurities in the gasses used for growth was specified to be at a level of $10 \mathrm{ppm}$. No additional gas purification was used in this experiment. Thus, we assumed that the residual concentration of nitrogen in growth plasma did not exceed $10 \mathrm{ppm}$. Nitrogen was added to the gas mixture in amounts: $0 \mathrm{ppm}$ (first layer), $14 \mathrm{ppm}$ (second layer), $27 \mathrm{ppm}$ (third layer), $54 \mathrm{ppm}$ (fourth layer), $82 \mathrm{ppm}$ (fifth layer) and $122 \mathrm{ppm}$ (sixth layer). Correspondingly, the atomic nitrogen content relative to the atomic carbon content in gas was about 240, 570, 880, 1520, 2130 and 3140 ppm respectively.

After growth, diamond block was cut in several plates along a (100) plane perpendicular to the growth surface (cross-sectional cut). Each plate was mechanically polished on both sides to a thickness of $0.3 \mathrm{~mm}$. In this research we used three plates \#5293-4, \#5293-5 and \#5293-6.

The plate \#5293-4 was irradiated with $1 \mathrm{MeV}$ electrons at a dose of $10^{18} \mathrm{~cm}^{-2}$ and subsequently annealed in vacuum at a temperature of $800{ }^{\circ} \mathrm{C}$ for an hour. This irradiation-annealing treatment was used in order to create additional $\mathrm{NV}$ defects and to make the nitrogen present in diamond easily detectable visually via characteristic pink color (Fig. 1a) and spectroscopically via luminescence and absorption of NV centers (to be published elsewhere).

Total concentration of nitrogen was analyzed with secondary ion mass spectrometry (SIMS) in the plate \#5293-4 at 30 points across all growth layers from the substrate to the top growth surface (Fig. 1a). The first two points were chosen on the substrate. Two other plates \#5293-5 and \#5293-6 were used for visual examination, for analysis of birefringence, and for the measurements of optical absorption and Raman scattering (Figs. 1b, 3a). 

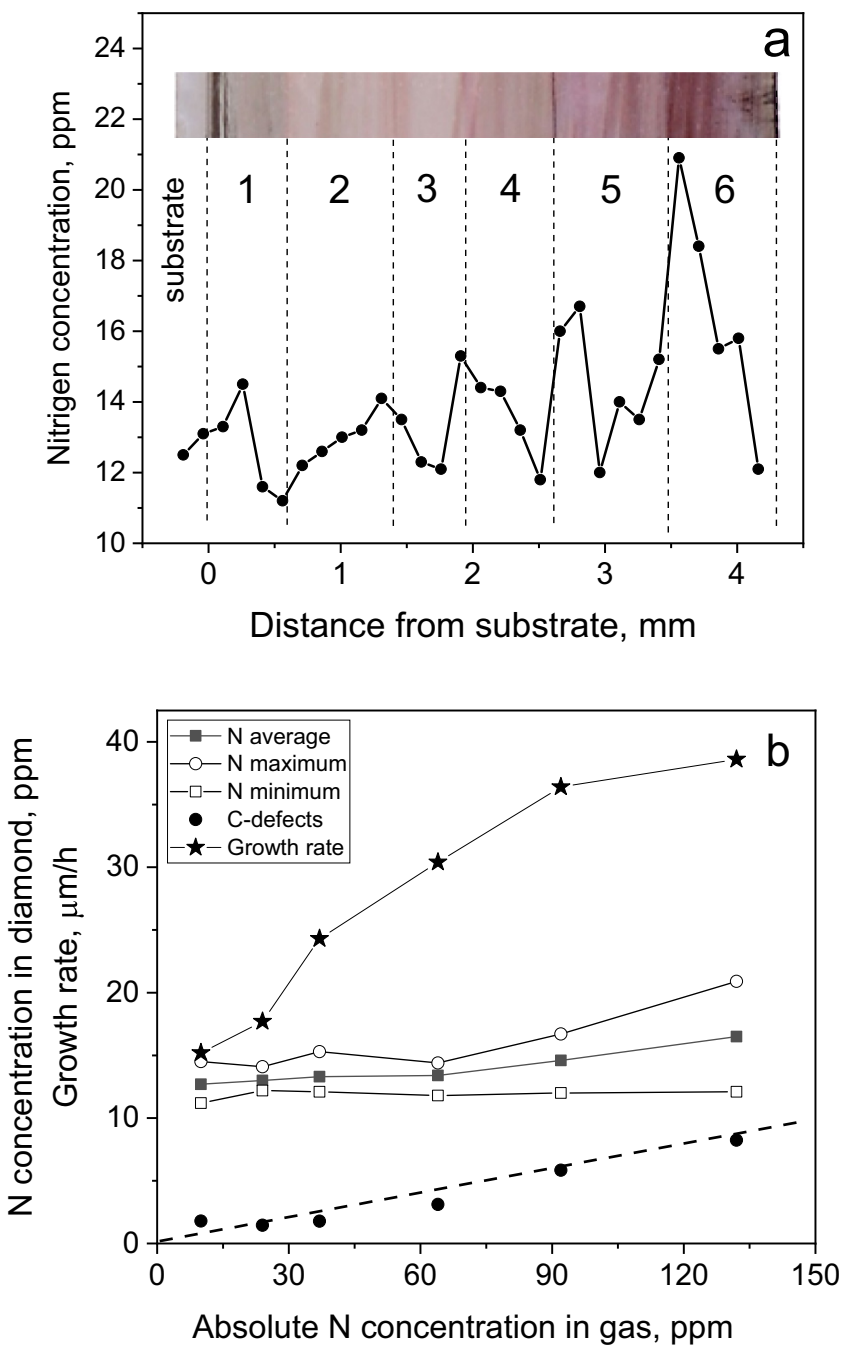

Fig. 2. (a) Nitrogen concentration measured with SIMS is compared with the optical image of the plate \#5293-4. The growth layers are labeled with numbers 1 to 6 . Dashed vertical lines show positions of the interlayer boundaries. (b) Maximum, minimum and average concentration of nitrogen measured with SIMS, concentration of C-defects measured in absorption and the growth rate for each layer are plotted versus absolute nitrogen concentration in growth gas.

The SIMS measurements were performed with a CAMECA IMS 1280 in the WiscSIMS lab, University of Wisconsin. A $2.5 \mathrm{nA}$ current of $\mathrm{Cs}^{+}$ ions was used as the primary beam. The spot size of the ion beam on sample surface was $10 \times 12 \mu \mathrm{m}^{2}$ with oval shape. Count rates of secondary ions of ${ }^{24}\left[{ }^{12} \mathrm{C}^{12} \mathrm{C}\right]$ and ${ }^{26}\left[{ }^{12} \mathrm{C}^{14} \mathrm{~N}\right]$ were measured using two Faraday cups simultaneously. More details of the analytical setting can be found in [11]. The use of diamond samples with known nitrogen concentrations (Tingting Gu, personal communication, Jan, 2019; nitrogen concentrations in diamond samples A5359, A5361 and A0927 were determined by SIMS at Canadian Center for Isotopic Microanalysis) as the standards for nitrogen concentration and comparison of the nitrogen concentration measured with SIMS and the nitrogen concentration measured with FTIR and UV absorption spectroscopies (to be published elsewhere) allowed us to assume that the uncertainty in absolute nitrogen concentration did not exceed $10 \mathrm{ppm}$. The relative differences in nitrogen concentration measured with SIMS were quite precise.

Raman spectra were measured at room temperature using the spectrometer Nanofinder High End (LOTIS TII Tokyo Instruments) combined with a 3D scanning confocal microscope. Most measurements were performed with laser excitation at wavelengths $354.8 \mathrm{~nm}$ (solid state laser). For some measurements, the excitation at wavelengths 473 and $532 \mathrm{~nm}$ was used. In most cases the Raman spectra were measured with a low spectral resolution of $5 \mathrm{~cm}^{-1}$. This resolution was sufficient for correct measurements of spectral shape and position of broad Raman features like second-order scattering from diamond and scattering from non-diamond carbon inclusions. The measurements of the position and width of narrow diamond Raman line at $1332 \mathrm{~cm}^{-1}$ were performed with resolution better than $0.25 \mathrm{~cm}^{-1}$. The intensity of the Raman scattering from diamond was evaluated either by the peak intensity of the second order feature at $2480 \mathrm{~cm}^{-1}$ (for low resolution spectra), or as a product of peak intensity and width (FWHM) of the line at $1332 \mathrm{~cm}^{-1}$ (for high resolution spectra). The measurements were performed at many points along the red lines shown in Fig. $1 \mathrm{~b}$.

$\mathrm{UV}-\mathrm{Vis}$ absorption spectra were measured at room temperature in the spectral range from 200 to $900 \mathrm{~nm}$ using a Cary 300 Bio (Agilent Technologies) spectrometer. The measured areas were rectangles of size about $0.3 \times 1.2 \mathrm{~mm}^{2}$ (see Fig. 1b).

\section{Experimental results}

\subsection{Visual imaging}

In the as-grown state, the plates reveal grayish-brown color in all growth layers (Fig. 1). After irradiation and annealing, plate \#5293-4 shows a distinctive pink color (Fig. 1a). All interlayer boundaries are visually discernable. The boundaries between the substrate and the 1st growth layer (boundary 0-1) and between 4th and 5th layers (boundary 4-5) are very pronounced and seen as dark sharp lines. The boundary $0-1$ has fine complex structure revealing the presence of several thin layers. The other boundaries are seen as abrupt changes in the color distribution and in the shape of color patterning. The black color of the boundaries $0-1$ and 4-5 evidences the formation of non-diamond carbon. Although the conditions of the sample surface preparation prior to growth and the growth regimes were nominally kept identical, these two boundaries turned out to be much more defective.

The distribution of gray and brown color is very non-uniform revealing irregular diffused striations and patches. On average, the intensity of brown color (as well as the intensity of pink color in the plate \#5293-4) increases from the 1st layer to the 6th layer as the concentration of nitrogen in the growth gas goes up. The striations are not ideally parallel and straight. However, most of them reveal a preferential orientation at an angle of $12^{\circ}$ with respect to the interlayer boundaries. Some striations are oriented horizontally parallel to the growth interfaces. These striations are labeled with (HS) in Fig. 1a.

Comparing the images of the plates \#5293-5 and \#5293-4 (Fig. 1), it is seen that the distribution of brown color follows the distribution of pink color. Some areas have distinctive gray color component in both plates. This gray color is not so obvious in the areas with dominating brown and pink colors. Interestingly, the nominally undoped 1st layer has the deepest gray color and it does not reveal noticeable pink color in plate \#5293-4.

\subsection{SIMS measurements}

The distribution of nitrogen concentration measured by SIMS is shown in Fig. 2. Although there is a general tendency for the nitrogen content in the diamond to rise with the increase in the nitrogen concentration in the growth gas, its distribution is highly nonuniform even within one growth layer. For instance, the variations in the nitrogen concentration in the 6th layer are from 11 to 21 ppm (Fig. 2a). There is a visual correlation of intensity of brown (and related pink) color and the nitrogen concentration measured with SIMS (Fig. 2a). The areas of intense brown/pink color have on average higher nitrogen content, whereas the areas of low intensity of color reveal lower levels of nitrogen doping. All interlayer boundaries grown with nitrogen content in gas above $20 \mathrm{ppm}$ reveal maxima in nitrogen concentration. 

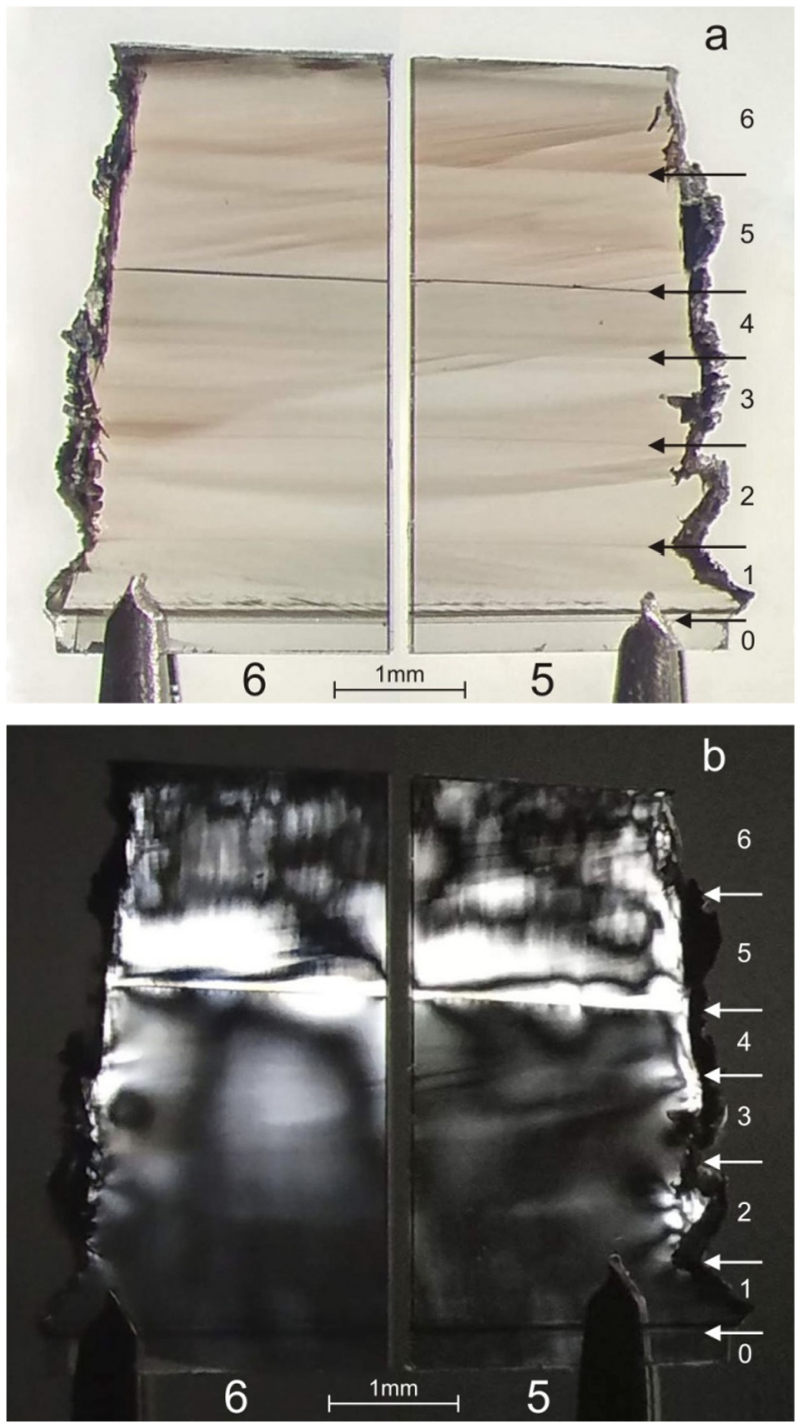

Fig. 3. Optical images (a) and birefringence patterns (b) of plates \#5293-5 and \#5293-6. The arrows in images show positions of interlayer boundaries.

In Fig. $2 \mathrm{~b}$, the growth rate of diamond is compared with the nitrogen concentration in diamond. It is seen that the addition of nitrogen to growth gas substantially stimulates growth rate of diamond. In contrast, the nitrogen concentration trapped in diamond weakly depends on the nitrogen concentration in gas. For instance, the minimum concentration measured with SIMS in each growth layer remains almost constant at a level of $10 \mathrm{ppm}$ over the whole sample.

\subsection{Birefringence}

Fig. 3 shows the optical images and birefringence patterns of the plates \#5293-5 and \#5293-6. The distribution of the anomalous birefringence is very non-uniform. On average, the anomalous birefringence in the first four layers is much weaker than in the layers 5 and 6 . It is especially strong in the 5th layer close to the boundary 4-5. Interestingly, the boundary $0-1$, which is black and obviously very defective, did not cause noticeable stress in the first growth layer.

Layer 5 exhibits the strongest anomalous birefringence (the brightest areas in Fig. 3b). It begins sharply at the boundary with the 4th layer and, as the 5th layer grows, the birefringence intensity goes down. The subsequent 6 th layer shows weaker anomalous birefringence than the 5th layer. All interlayer boundaries, with exception of the boundary 4-5, do not reveal strong contrast in the birefringence
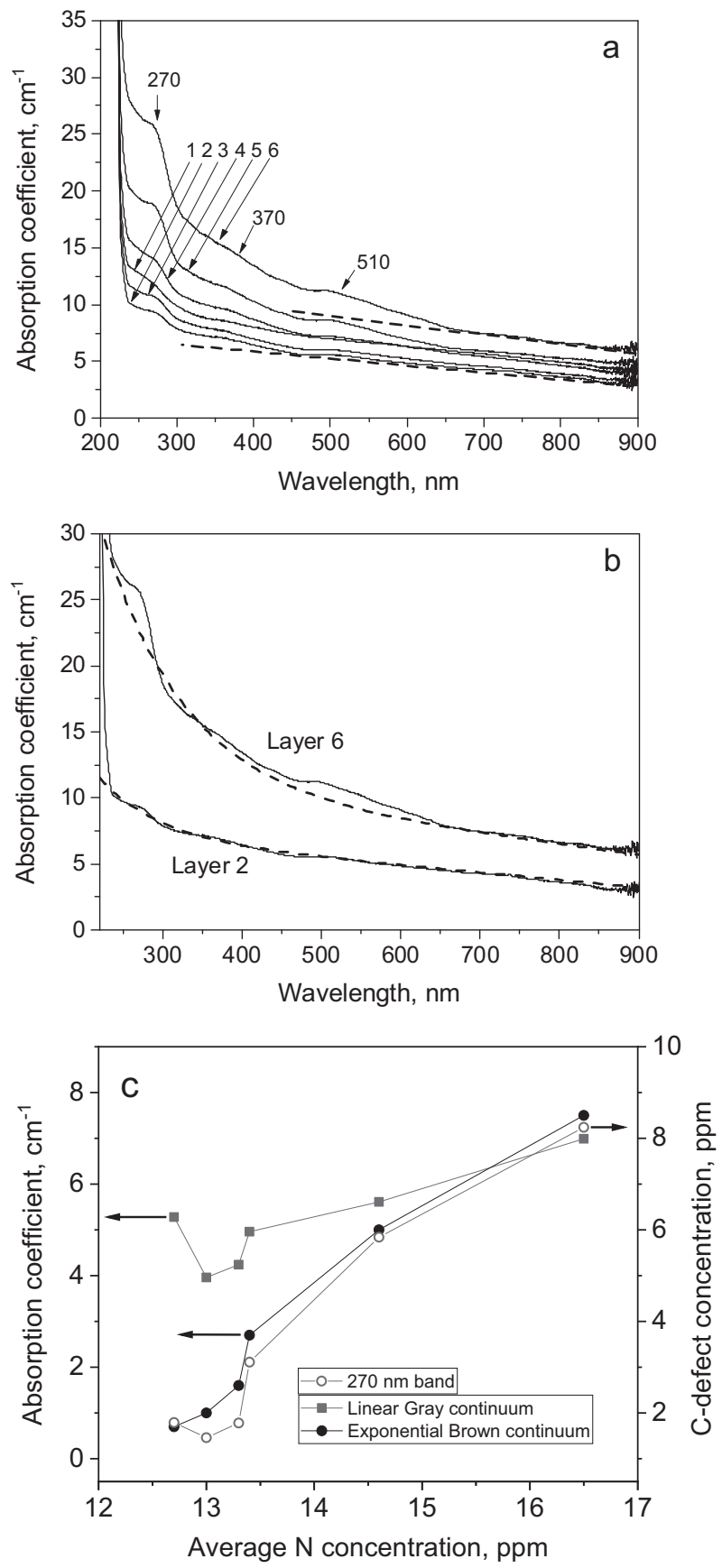

Fig. 4. (a) Absorption spectra of layers 1 to 6 . Dashed lines show linear absorption continua for layers 2 and 6. (b) Experimental absorption spectra (solid lines) approximated by functions $\mathrm{y}(\mathrm{x})=[(12.7-0.0078 * \mathrm{x})+180 * \exp$ $(-\mathrm{x} / \mathrm{100})]$ for layer 6 and by function $\mathrm{y}$ $(\mathrm{x})=[(7.85-0.0055 * \mathrm{x})+85 * \exp (-\mathrm{x} / 75)]$ for layer 2 (dashed lines). (c) Absorption intensities at wavelength $800 \mathrm{~nm}$ (intensity of the linear continuum; gray color component), at wavelength $300 \mathrm{~nm}$ (intensity of the exponential continuum with the intensity of gray continuum subtracted; brown color component), and concentration of C-defects (calculated from intensity of $270 \mathrm{~nm}$ band) plotted versus average concentration of nitrogen measured with SIMS.

intensity.

Birefringence reveals a striation pattern almost all over the sample. In most cases, these striations do not follow the directions of interlayer boundaries, but the directions of the color striations. Although the intensity of the anomalous birefringence is the strongest in the layers 5 and 6 , where the brown/gray color is the most intense, and although 
the birefringence follows the pattern of the brown/gray color, there is no perfect correlation between them. There are areas with intense color but low anomalous birefringence while there are low-colored areas exhibiting strong anomalous birefringence.

\subsection{UV-Vis absorption}

Absorption spectra measured as an average over each growth layer are shown in Fig. 4a. The spectra have a similar shape for all layers: a continuum of rising intensity towards shorter wavelengths. The experimental spectra can be well approximated by sum of linear and exponential functions. In Fig. $4 \mathrm{~b}$, this approximation is shown for absorption spectra of layers 2 and 6 with functions: $y$ $(\mathrm{x})=[(12.7-0.0078 * \mathrm{x})+180 * \exp (-\mathrm{x} / 100)]$ and $\mathrm{y}$ $(\mathrm{x})=[(7.85-0.0055 * \mathrm{x})+85 * \exp (-\mathrm{x} / 75)]$ respectively. Thus, the long wavelength part of the spectra can be presented by a straight line (Fig. 4a), while at wavelengths shorter than $650 \mathrm{~nm}$, the fast-rising absorption can be described as exponential. Intensity of both continua increases with nitrogen concentration in diamond (Fig. 4c).

On the background of these continua, the broad bands at wavelengths 270,370 and $510 \mathrm{~nm}$ are observed. The intensity of the $270 \mathrm{~nm}$ band correlates with the intensity of the exponential absorption continuum (Fig. 4c).

The intensity of the exponential absorption continuum increases with nitrogen doping faster than that of the long wavelength straightline absorption. The absorption spectra of the layers 1 and 2 in the whole visible spectral range appear to be composed of only the straightline continuum. The color of these two layers is gray without visible brown component. Thus, we ascribe the straight-line absorption continuum to the observed gray color and term it the gray absorption continuum. The color of layer 6 is obviously brown and the presence of the fast-rising absorption continuum in its spectrum is significant. Therefore, we ascribe the exponentially-rising absorption to the brown color and term it the brown absorption continuum. It is seen that the brown color becomes progressively dominating over gray color with the increase in nitrogen concentration. In the low-nitrogen layers, the dominating color is gray. This difference is clearly seen when comparing the colors of the layers 1 and 6 in Figs. 1 and $3 a$.

\subsection{Raman measurements}

Raman spectra measured at many points overall growth layers are qualitatively similar (Fig. 5). Very strong first order Raman line at $1332 \mathrm{~cm}^{-1}$ and pronounced band of two-phonon scattering in the range 2200 to $2660 \mathrm{~cm}^{-1}$ dominate the spectra. Minor broad lines at wavenumbers about 1550, 1590, 2865 and $3100 \mathrm{~cm}^{-1}$ are also present. Spectral widths of these lines are about $35,35,65$ and $95 \mathrm{~cm}^{-1}$ respectively. A weak feature at a wavenumber $1420 \mathrm{~cm}^{-1}$ is always seen as a little shoulder on the wing of the major $1332 \mathrm{~cm}^{-1}$ line. Because of the strong overlapping with the diamond Raman line, we can make only an approximate estimation of its width which amounts to about $20 \mathrm{~cm}^{-1}$. As these features are detected in spectra taken with different excitation wavelengths, we are confident assigning them to Raman scattering. Spectral position of these lines can vary up to $\pm 10 \mathrm{~cm}^{-1}$ when measured in different locations.

The lines 1550, 1590, 2865 and $3100 \mathrm{~cm}^{-1}$ have elevated intensity in the spectra of the defective interlayer boundaries $0-1$ and 4-5 (Fig. 5c). However, they can be also relatively intense in the spectra of visually inclusion-free regions. Interestingly, the high-quality interlayer boundary $3-4$, which is hardly visible in microscope, reveals these Raman features too (see Fig. 5b).

Intensities of the lines 1550, 2865 and $3100 \mathrm{~cm}^{-1}$ strongly fluctuate from point to point. This sporadic behavior is demonstrated in Fig. 6a for the line at $1550 \mathrm{~cm}^{-1}$. Notwithstanding the fluctuations, average intensity of the line $1550 \mathrm{~cm}^{-1}$ slightly increases from layer 1 to layer 6. In contrast, the line $1590 \mathrm{~cm}^{-1}$ is clearly seen only in spectra of the
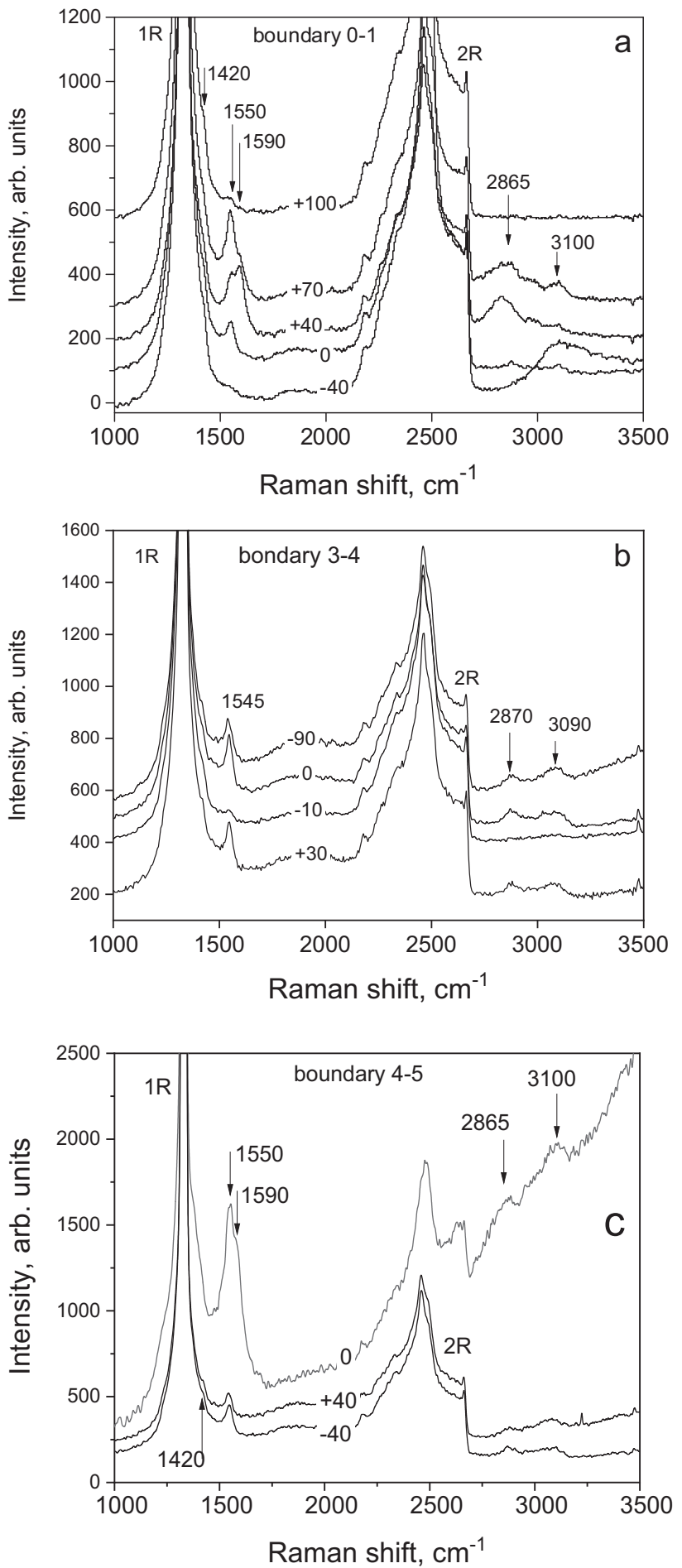

Fig. 5. Selected Raman spectra (excitation with $354.8 \mathrm{~nm}$ laser) measured close to interlayer boundaries. (a) Boundary 0-1: in substrate, $40 \mu \mathrm{m}$ away from the boundary $(-40)$; in the 1st layer, $40 \mu \mathrm{m}(+40), 70 \mu \mathrm{m}(+70)$ and $100 \mu \mathrm{m}$ $(+100)$ away from the boundary. (b) Boundary 3-4: in the 3rd layer, $10 \mu \mathrm{m}$ $(-10)$ and $90 \mu \mathrm{m}(-90)$ away from the boundary and in the 4th layer $30 \mu \mathrm{m}$ $(+30)$ away from the boundary. (c) Boundary 4-5: in the 4th layer, $40 \mu \mathrm{m}$ away from the boundary $(-40)$ and in the 5 th layer, $40 \mu \mathrm{m}$ away from the boundary $(+40)$. The spectra labeled with 0 were taken at the boundaries. Intensity of all spectra is adjusted to equal intensity of the diamond Raman line at $1332 \mathrm{~cm}^{-1}$. Spectra are shifted vertically for clarity. 

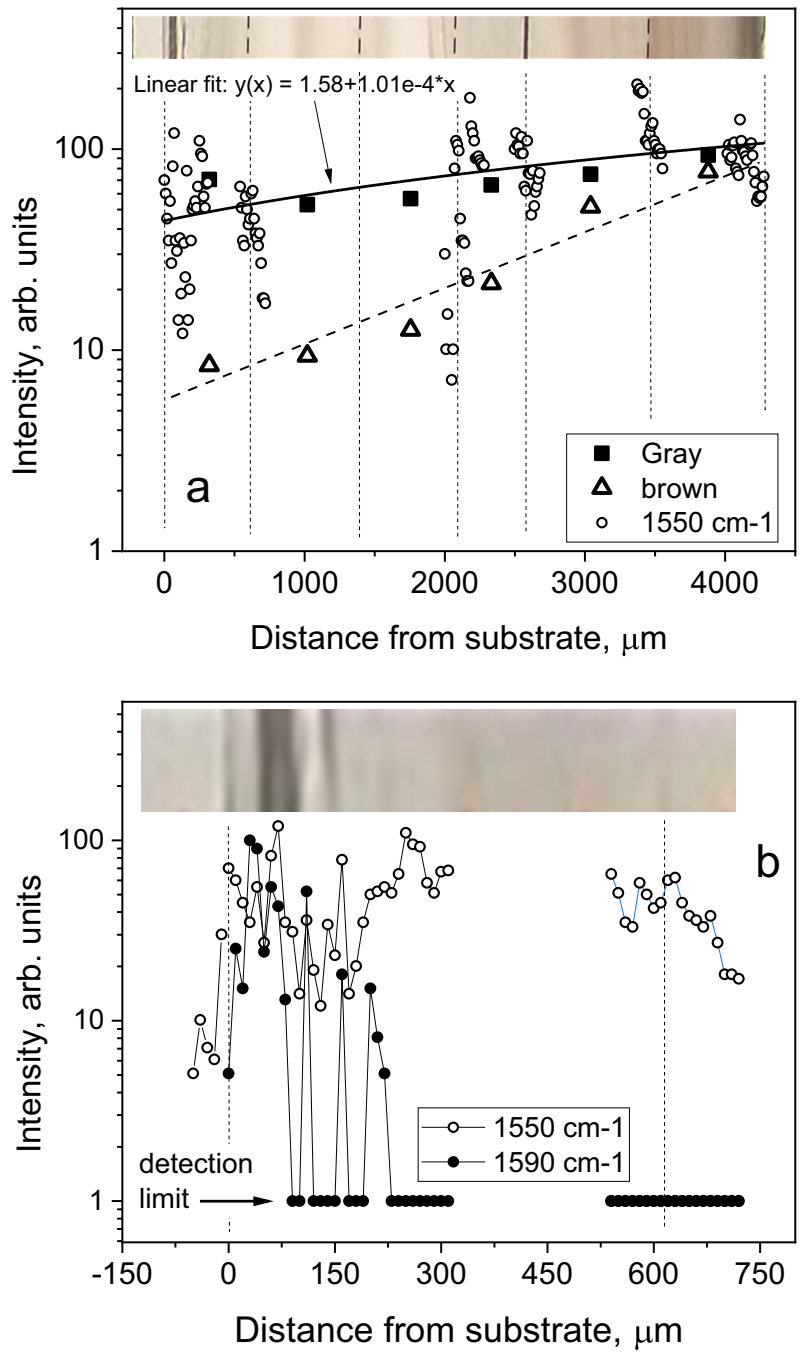

Fig. 6. (a) The distribution of the intensity of the Raman line $1550 \mathrm{~cm}^{-1}$ (open circles) over six layers is compared with the intensities of gray (solid squares) and brown (open triangles) absorption continua. Solid line shows the linear fit of the intensity distribution of the line $1550 \mathrm{~cm}^{-1}$ (Pearson's correlation factor of 0.53 ). The dashed line shows the tendency in the intensity change of the brown absorption continuum. (b) The detailed distribution of intensities of the Raman lines $1550 \mathrm{~cm}^{-1}$ (open circles) and $1590 \mathrm{~cm}^{-1}$ (solid circles) over the (0-1) and (1-2) boundaries. The vertical dashed lines show the positions of the interlayer boundaries.

most defective boundaries $0-1$ and $4-5$ and in the areas of the first layer where black inclusions are pronounced (Fig. 6b). Its intensity is at the detection limit in the spectra taken from the bulk of the layers and from the boundaries 1-2, 2-3, 3-4 and 5-6 where no visible black inclusions are present.

The comparison of the intensity of the gray absorption continuum with the average intensity of the $1550 \mathrm{~cm}^{-1}$ Raman line reveals their correlation (Fig. 6a). The increase in intensity of the $1550 \mathrm{~cm}^{-1}$ line can be approximated by a linear function with correlation factor of 0.53 (shown with solid line in Fig. 6a). This linear approximation well follows the increase in intensity of the gray absorption continuum. No correlation is seen between the intensities of the brown absorption continuum and the $1550 \mathrm{~cm}^{-1}$ line. Other Raman lines do not show reasonable correlation neither with gray, nor with brown absorption continua. See, for instance, the intensity distribution of $1590 \mathrm{~cm}^{-1}$ line in Fig. $6 \mathrm{~b}$. The $1590 \mathrm{~cm}^{-1}$ line is not detectable even at the end of the 6th layer, which has the strongest brown/gray color.

The intensity, spectral width and spectral position of the diamond
Raman line $1332 \mathrm{~cm}^{-1}$ measured in many places all over the sample is shown in Fig. 7. The measurements were performed especially thoroughly close to the interlayer boundaries.

The intensity of the diamond Raman line, measured as the product of its peak intensity and its width, reduces at the interlayer boundaries (Fig. 7a, d). This reduction is particularly strong at the most defective boundaries $0-1$ and $4-5$. The minimum intensity of the Raman line is found at the interlayer boundary $4-5$, which is probably the most defective region of the sample.

In general, the line $1332 \mathrm{~cm}^{-1}$ broadens at the interlayer boundaries (Fig. 7b, e). Surprisingly, no broadening is observed at the boundary $0-1$, which is visually very defective. In contrast, the boundary $4-5$, which is visually as defective as the boundary $0-1$, reveals very strong broadening of the Raman line. The broadening at the boundary $4-5$ is especially pronounced on the side of the 5 th layer. The spectral position of the diamond Raman line is stable all over the sample except at the boundary 4-5, where it shows some oscillatory behavior. The spectral position shifts interchangeably towards greater and smaller wavenumbers when moving across the boundary 4-5 and into the 6th layer. Detailed distributions of the intensity, spectral width and spectral position of the Raman line across the boundary 4-5 are shown in Fig. $7 d$, e, f. It is seen that the spectral shift of the line is stronger on the side of layer 5 than on the side of layer 4 (Fig. 7f).

\section{Discussion}

\subsection{Brown color}

Gray and brown colors are the main color components of as-grown sample. We attribute these colors to the gray and brown absorption continua described above. The dominating pink color of the irradiated and annealed plate is due to absorption on negatively charged NV defects $\left(\mathrm{NV}^{-}\right.$centers). The fact that nitrogen-doped CVD diamonds acquire pink color after irradiation followed by annealing at moderate temperatures (e.g. after electron irradiation at a dose of $10^{18} \mathrm{~cm}^{-2}$ followed by heating at $1000{ }^{\circ} \mathrm{C}$ ) is well-known [9,12-15]. Therefore, there is no need to present and discuss in this communication the absorption spectra of pink areas. There is a visual correlation between the intensities of the brown and pink colors suggesting that they both relate to nitrogen doping (compare Figs. 2a and 3). This observation is also supported by the SIMS (Fig. 2a) and absorption (Fig. 4c) measurements.

A very pronounced feature of the color distribution is the striations. Strong non-uniformity of the distribution of the brown color is typical for CVD diamond (e.g. [12,16]). The origin of formation of these striations has not been established conclusively yet. However, the model based on growth at step edges and terraces [12] is very plausible. Our preliminary measurements of the orientation and shape of the terraces suggest that the terrace edges form mostly along (111) planes. Edges of some striations are oriented along (110) plane (to be published). In our sample plates cut along (100) plane, most of striations run at an angle $12^{\circ}$ with respect to the growth surface. We assume that these striations have been formed by (111)-edged terraces. In order to make this slope, the growth rate of (111)-oriented edge, and correspondingly the horizontal propagation of terrace along $\langle 100\rangle$ direction, must be about 7 times faster than the vertical growth of the (100)oriented terrace flat. The relatively high growth temperature used in our growth is probably one of the reasons for the fast growth of the (111)-oriented facets $[17,18]$. CVD diamonds grown on (111)-oriented substrates are more defective than the diamond grown on (100)-oriented substrates ([17] and references therein). These defects are likely the cause of the brown color of the striations. Incorporation of nitrogen during diamond growth also occurs preferentially in (111)-oriented sectors [19]. Thus, the striations are expected to be more defective and contain higher concentration of nitrogen.

In some CVD diamonds (e.g. [14,20]) and even within one sample the brown striations can have different orientations. For instance, a few 

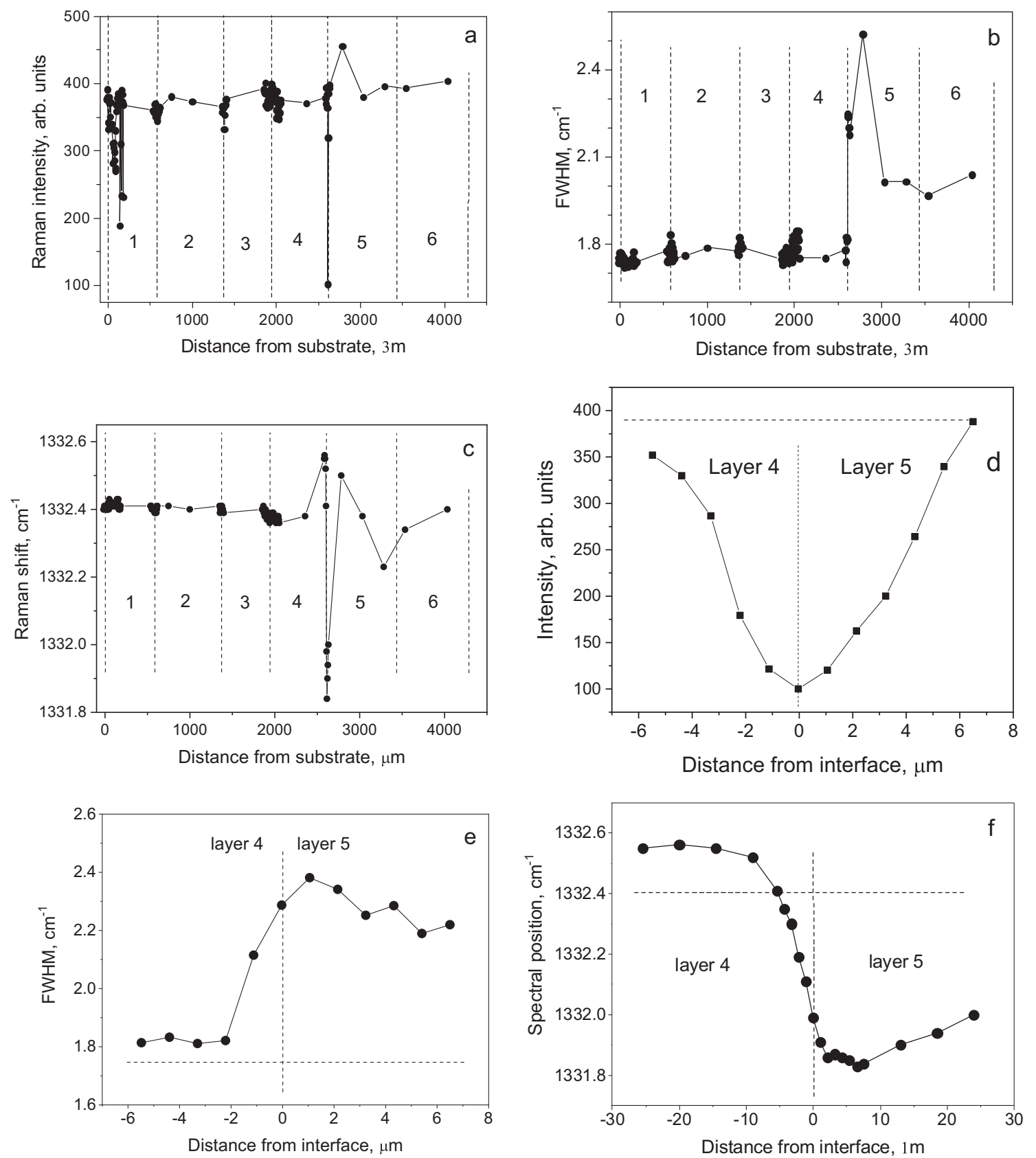

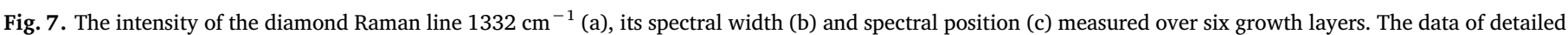

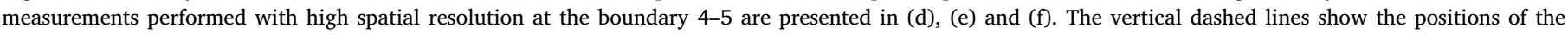

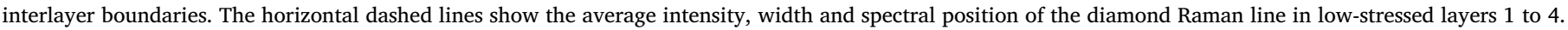

striations in our plates are oriented parallel with respect to the substrate surface (horizontal striations) (Figs. 1,3). We found that in our case these striations are cross-sectional images of the $12^{\circ}$-angled striations viewed in (100) plane cut perpendicular to the direction of their propagation.

One could think of other possible reasons of formation of color striations like the fluctuations in the flux of nitrogen impurities to the growth surface, or the variations in temperature of the growing surface. SIMS measurements show strong fluctuations in the rate of nitrogen incorporation during growth (Fig. 2a). These fluctuations cannot be explained by the fluctuations of the nitrogen concentration in the gas reactants. The reactor operation and the flow of gas components were very stable during growth. Temperature variations seem to be a more plausible explanation of the formation of striations [14]. However, in our experiment, the temperature variations of the growth surface did not exceed $40{ }^{\circ} \mathrm{C}$. Could these little temperature variations result in such large fluctuations in nitrogen content and in color?

The brown striations of CVD diamonds are similar in appearance to the brown striations of natural diamonds. Correspondingly, the optical absorption spectra of brown CVD diamonds are similar to the spectra of natural brown diamonds (e.g. $[12,21,22]$ ) (Fig. 8a). In both cases, the brown absorption continua can be well fitted by exponential functions. However, despite the similarity of the optical features, the thermal stability of the defects causing brown color in CVD diamonds is lower than in natural diamond [13]. Because of this difference, the model of globular vacancy clusters commonly used for the explanation of brown color in natural diamonds [23], has not been fully accepted for CVD diamonds. Formation of vacancy discs, which have lower thermal 

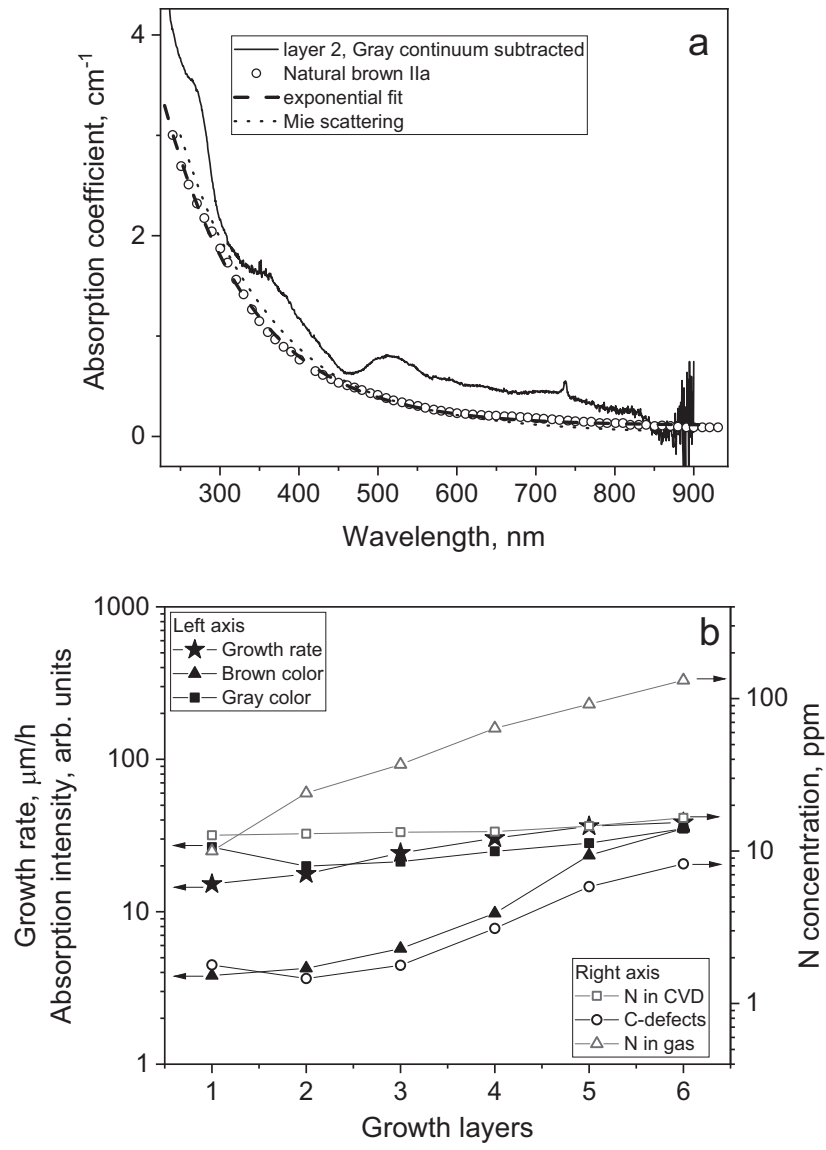

Fig. 8. (a) A comparison is shown of the brown absorption continuum of layer 2 with the absorption continuum of brown natural diamond taken from [22]. The dashed curve shows the exponential fitting curve $y(x)=3.08-2.97\{1-\exp$ $[-(x-237.7) / 111]\}$. The dotted line shows the intensity of the simulated Mie scattering. (b) The change in intensity of brown and gray absorption continua from layer 1 to layer 6 is compared with the growth rate, average nitrogen concentration measured by SIMS and the concentration of C-defects measured in absorption.

stability, was proposed in [24,25] to account for the color of brown CVD diamonds. We follow the vacancy cluster model though without going into details concerning the shape of these clusters. Vacancy clusters of any shape and size have no definite atomic structure and consequently no definite electronic structure. Thus, any vacancy clusters should generate broad featureless continua in optical absorption like those shown in Figs. 4 and 8. In CVD diamonds, which grow at lower temperatures and for much shorter time than natural diamonds, the vacancy clusters are expected to be smaller. We assume that small vacancy clusters, irrespective of their shape, have lower temperature stability than large ones.

Although we assume that the optical absorption of vacancy clusters is the main origin of the brown absorption continuum, the contribution of light scattering could be substantial too. For clusters much smaller that the wavelength of visible light it is Rayleigh scattering, while for larger clusters (nanocavities) Mie scattering [26] is the probable mechanism of scattering. We performed a simulation of the intensity of Mie scattering [27] on spherical vacancy cavities in a diamond matrix for different cavity sizes and concentrations. The simulation for $40 \mathrm{~nm}$ cavities with a concentration of $10^{12} \mathrm{~cm}^{-3}$ resulted in a surprisingly good fit with the experimental absorption spectrum of natural diamond (Fig. 8a). In spite of this result we do not suppose that Mie scattering is the main contributor to brown absorption continuum. The fact that the brown absorption continuum is detected by photothermal deflection spectrometry [28] suggests that optical absorption is dominant contributor. However, the contribution of light scattering should not be ruled out as negligible.

We have found that the growth rate of our CVD diamond did not correlate well with the generation of brown color (Fig. 8b). The intensity of the brown color increases much faster than the growth rate. We can conclude that the growth rate and the intensity of brown color are not closely related. In support of this conclusion we have examples of brownish CVD diamonds grown at a low rate below $10 \mu \mathrm{m} / \mathrm{h}$ and near-colorless CVD diamonds grown at a rate as high as $50 \mu \mathrm{m} / \mathrm{h}$.

With the addition of nitrogen, the intensity of brown color goes up (Fig. 8b). However, not all nitrogen trapped into diamond influences the brown color equally. The intensity of the brown absorption continuum correlates well only with the concentration of nitrogen C-defects, but not with the total nitrogen concentration measured by SIMS. This observation supports the findings of Hainschwang and co-authors [29] that C-defects are one of the main contributors to brown color of natural brown type Ib diamonds. The brown color intensity also follows well the nitrogen concentration in growth gas. We assume that the influence of nitrogen on the generation of brown color occurs via the change in morphology of growing surface. It is well known that the growth morphology of CVD diamond strongly depends on the presence of nitrogen in growth gas. Thus, we may assume that the nitrogen-induced change in growth morphology favorable for terraced growth is a factor of the formation of vacancy clusters, generation of brown color and the stimulation of the capture of isolated nitrogen atoms from plasma.

The optical absorption bands at wavelengths 270, 370 and $510 \mathrm{~nm}$ (Fig. 4a) are ascribed to nitrogen-containing defects [5,30,31]. These bands are observed in absorption spectra of most CVD and natural diamonds. The $510 \mathrm{~nm}$ band $(520 \mathrm{~nm}$ band in some publications, e.g. $[12,15])$ is likely an analog of the Pink band (a broad band with maximum at wavelength about $550 \mathrm{~nm}$ ), which is characteristic of absorption spectra of natural pink and brown diamonds [13,22,31,32]. The $510 \mathrm{~nm}$ band can be enhanced by high temperature annealing (to be published) in the same way as it is known for the Pink band in natural type IIa brown diamonds [22]. Although the nitrogen-related bands 270,370 and $510 \mathrm{~nm}$ are not the direct constituents of brown absorption continuum, their presence may substantially contribute to the intensity of brown color in nitrogen-doped CVD diamonds.

The relation between nitrogen doping and brown color is not specific to CVD diamonds only. A vast majority of natural diamonds are brown and a vast majority of natural diamonds are nitrogen-doped. Are these very well-known facts related? Of course, vacancy clusters can be generated by mechanisms other than nitrogen doping. Plastic deformation, drift of dislocations and irradiation are the natural processes very effective in creation of vacancies. However, there are many examples of natural brown diamonds which do not show any evidence of these processes. Many non-deformed and non-irradiated natural diamonds are nitrogen-containing and brown. Could it be that the presence of nitrogen in the growth medium of these diamonds and the nitrogen doping were the factors which stimulated their brown color? For natural diamonds, the percentage of brown stones is higher for type I diamonds than type IIa diamonds. Surface morphology of type I natural diamonds is better than the surface morphology of type IIa diamonds. Are these facts not something resembling the nitrogen-free and nitrogen-doped CVD diamonds? CVD diamond growers know that addition of nitrogen to growth gas improves the morphology of growth. A supposition can be made that for natural diamonds, like for CVD diamonds, nitrogen doping makes growth morphology smoother, reduces internal stress but, unfortunately, stimulates the generation of brown color.

\subsection{Gray color}

A distinctive feature of the absorption spectra shown in Fig. 4a is the gray absorption continuum, which is the cause of gray color. We ascribe 

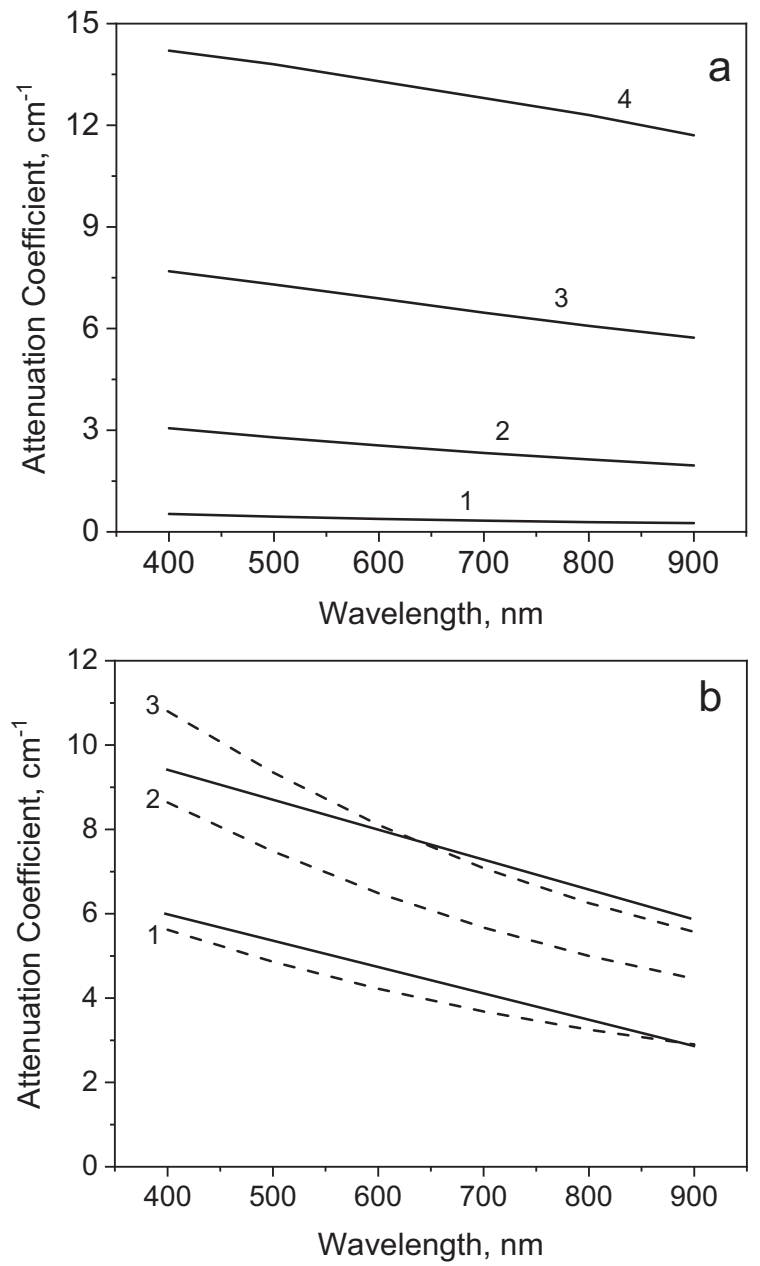

Fig. 9. The spectral dependence of the intensity of Mie scattering from spherical graphitic nanoclusters in diamond matrix. (a) The scattering intensity from clusters of size $50 \mathrm{~nm}$ (1), $100 \mathrm{~nm}$ (2), $150 \mathrm{~nm}$ (3) and $200 \mathrm{~nm}$ (4) for a concentration of $2 \times 10^{10} \mathrm{~cm}^{-3}$. (b) The scattering intensity from $60 \mathrm{~nm}$ graphitic clusters for concentrations $1.3 \times 10^{11} \mathrm{~cm}^{-3}(1), 2 \times 10^{11} \mathrm{~cm}^{-3}$ (2) and $2.5 \times 10^{11} \mathrm{~cm}^{-3}(3)$ are shown with dashed lines. Solid lines show experimental gray absorption continua for layers 2 (lower line) and 6 (upper line) approximated by straight lines.

this continuum to $\mathrm{sp}^{2}$-bonded extended defects. These defects, in contrast to point defects, do not have can have a diverse range of atomic and electronic structure. Therefore, they do not result in a specific structured absorption with distinctive features related to discrete electron and electron-vibrational transitions. Clusters of pi-bonded $\left(\mathrm{sp}^{2}\right)$ carbon atoms are a likely model of these defects. Since they are not seen in optical microscope with $\times 2000$ magnification, we will call them "carbon nanoclusters".

Most carbon particles are not transparent to visible light. So, light scattering from them could be substantial. For carbon nanoclusters of size comparable with wavelength of visible light, Mie scattering (see e.g. [26]) dominates over Raleigh scattering. We tested the idea of Mie light scattering by performing simulation assuming spherical shape of nanoclusters surrounded by diamond matrix (Fig. 9). Refractive index and extinction coefficient of graphite at wavelength $630 \mathrm{~nm}(2.7$ and 1.4 respectively) [33] were used for the calculations.

It is seen in Fig. 9a that the intensity of Mie scattering strongly depends on carbon cluster size. However, the spectral dependence is weak with almost linear increase as the light wavelength decreases from 900 to $300 \mathrm{~nm}$ (Fig. 9a). For small clusters of size $50 \mathrm{~nm}$ and below, the deviation of the spectral dependence from linear becomes stronger. This increasingly rising scattering at shorter wavelengths is probably due to contribution of Raleigh scattering. In Fig. 9b, the results of the simulation for $60 \mathrm{~nm}$ clusters with different concentrations are compared with the experimental gray absorption continua. The closest match is obtained for cluster concentrations in the range from $1.3 \times 10^{11} \mathrm{~cm}^{-3}$ to $2.5 \times 10^{11} \mathrm{~cm}^{-3}$. The results of simulation suggest that carbon nanoclusters can be a major component of the gray color in CVD diamond. Optical absorption may also contribute to the gray color as the carbon clusters likely contain extended $\mathrm{sp}^{2}$ bonded networks which absorb throughout the visible and UV range of the spectrum.

Hydrogen can also be a contributor to gray color component of CVD diamonds. Natural diamonds of gray color contain considerable amount of hydrogen in the form of optically active defects [34-36]. In nature, diamonds always grow in the presence of hydrocarbons (mainly methane and the products of its dissociation), carbon oxides, nitrogen hydrides, hydrogen and other hydrogen-containing molecules $[37,38]$. The crystallization of diamond occurs directly during dissociation of hydrocarbons into carbon and hydrogen in any growth medium: gaseous, silicate solutions, or metal melts. During diamond growth, hydrogen forms $\mathrm{CH}$ moieties on diamond surface, on the edges of graphite crystals, or bonds to $\mathrm{sp}^{2}$-bonded carbon defects. Thus, the amount of the hydrogen trapped into diamond substantially depends on the concentration of the graphitic or $\mathrm{sp}^{2}$-bonded inclusions and their size: the smaller the inclusions, the greater hydrogen concentration.

\subsection{Carbon nanoclusters}

Further evidence of the existence of non-diamond carbon inclusions are the Raman features at wavenumbers 1420, 1550, 1590, 2865 and $3100 \mathrm{~cm}^{-1}$. They are detected all over the sample. Although these lines are present together in many spectra, they do not show good correlation in intensity and therefore are attributed to different defects and/or inclusions of different carbon phases. Variations in the spectral positions of these lines suggest that the carbon inclusions vary in shape and size, and/or they experience strong perturbations in the stressed diamond lattice. Alternatively, a varying spectral position can be the result of a complex structure of Raman band composed of several strongly overlapping components.

The lines 1420, 1550, 1590, 2865 and $3100 \mathrm{~cm}^{-1}$ are not unique to nitrogen-doped CVD diamond. They are common features of Raman spectra of ion-implanted diamonds, low quality CVD diamonds grown by the torch-flame method, strongly deformed and damaged natural diamonds and diamond-like materials (e.g. [2,39-42]). The Lines 1550 and $1590 \mathrm{~cm}^{-1}$ are most likely the components of graphite G-band [43-45]. Traces of G-band are always observed in Raman spectra of asgrown CVD diamonds serving a reliable indicator of the presence of $\mathrm{sp}^{2}$ hybridized carbon inclusions.

In general, the intensity of the non-diamond Raman features, in particular the intensity of $1550 \mathrm{~cm}^{-1}$ line, follows the intensity of gray color (Fig. 6a). This correlation shows that the deterioration of color of as-grown CVD diamonds relates to the presence of non-diamond inclusions. The fact that non-diamond Raman features are present in spectra taken all over the sample including the defective interlayer boundaries, high-quality interfaces and perfect bulk is suggestive that it is not the quality of growth that primarily controls the concentration of the carbon nanoparticles, but the flux of these nanoparticles to the growing diamond surface.

The strong fluctuations of intensity of the non-diamond Raman lines shown in Fig. 6 are likely the consequence of low concentration of carbon nanoclusters and their random distribution. The simulation of Mie scattering suggests that the carbon nanoclusters in gray CVD diamond are tens of nanometers in size and their concentration is in the order of $10^{11}$ to $10^{12} \mathrm{~cm}^{-3}$. The average distance between the nanoclusters is about $5 \mu \mathrm{m}$. This value is comparable with the parameters of the spatial resolution of the confocal Raman spectrometer used (laser focal spot of $1 \mu \mathrm{m}$ and depth of focus of a few microns). Thus, the probabilities of probing the areas with one or a few nanoclusters or the 
areas with no nanoclusters are comparable.

The available experimental data do not give us enough evidence for a detailed comment on the atomic structure of the carbon nanoclusters. They could be heavy $\mathrm{C}_{n} \mathrm{H}_{z}$ species formed in the cooler outer regions of plasma [46-48], or solid carbon particles [49,50] which are not thermally desorbed or etched by atomic hydrogen. Alternatively, these carbon nanoclusters could be nanoscopic regions of non-diamond formations on the diamond surface. The dominating presence of $1550 \mathrm{~cm}^{-1}$ Raman line suggests that the aggregates with graphite-like atomic structure are likely candidates.

It is reported that the color grade of CVD diamond improves with the increase in the flow rate of growth gas in CVD reactor [46,47]. If the carbon nanoclusters are formed in plasma, then one might expect their concentration and size to depend on the gas residence time in the reactor chamber [50]. The faster flow of gas, the smaller size of solid carbon nanoclusters and the lesser their concentration at the diamond surface. The formation of carbon nanoclusters in the plasma is supported by the good correlation of the intensity of gray absorption continuum and the growth rate (Fig. 8b).

An unexpected result of our experiment is the very different quality of the interlayer boundaries. Although we followed the same procedure for the sample surface preparation before each growth and used the same growth conditions for all growth runs, the boundaries $0-1$ and 4-5 became conspicuously black with high amount of non-diamond carbon inclusions, while other interlayer boundaries became much lesser defective suggesting inclusion-free structure. We conclude that the quality of interlayer boundaries between CVD diamond layers is not determined only by the procedure of the surface preparation and the major growth parameters like the gas composition, gas pressure, plasma power density and substrate temperature, but by some other factors, which still remain unknown.

\subsection{Striations and stress}

The distribution of anomalous birefringence (Fig. 3) suggests that the stress in the first four layers is much weaker than in the layers 5 and 6 . Stress is especially strong in the 5th layer close to the highly defective boundary $4-5$. Interestingly, the boundary $0-1$ and the dark black regions of the layer 1 , which are seemingly as defective as the boundary 4-5, did not cause noticeable stress in the bulk of the first layer. In layer 5 , high lattice stress starts to form just at the interface 4-5. As the 5th layer grew, the stress went down. The following 6th layer grew with substantially less stress. These observations suggest that the presence of macroscopic defects on the growth surface do not necessarily prevent formation of low stressed diamond. A sort of "self-healing" takes place, which improves crystal lattice as the growth proceeds. We assume that the long-range crystallographic order of the substrate is more important requirement for the formation of continuous single crystal. Indeed, CVD growth occurs in many places over the substrate. These initial crystal islands become larger and, when perfectly aligned, merge into single crystal overgrowing local imperfections of the initial surface. This effect is especially evident in the first layer, which grew on visually very defective interface, but nevertheless did not collapse into polycrystal and even did not develop noticeable internal stress.

More detailed information on the distribution of stress is obtained from the measurements of intensity, spectral width and spectral position of diamond Raman line $1332 \mathrm{~cm}^{-1}$ (Fig. 7). The intensity of the line reduces at all interlayer boundaries (Fig. 7a, d) and in particular at the defective boundaries $0-1$ and 4-5. The lower Raman signal can be explained by the presence of high concentration of carbon nano-inclusions, which reduce optical transparency and also can reduce the lifetime of diamond phonons.

Spectral position of Raman line provides information on the hydrostatic component of crystal lattice deformation. Hydrostatic compression shifts diamond Raman line towards greater wavenumbers, while the lattice expansion causes shift towards lower wavenumbers. In the regions close to interlayer boundaries, two-dimensional (100) and equivalent uniaxial $\langle 100\rangle$ stress can be substantial too. Averaging the data obtained in numerous publications, see e.g. [51-56], we assume that the rate of the Raman line shift is about $\pm 2.5 \mathrm{~cm}^{-1} / \mathrm{GPa}$ (positive for compression and negative for expansion). Thus, the maximum stress of compression and expansion observed at the most stressed boundary 4-5 is about $0.4 \mathrm{GPa}$ and $1.5 \mathrm{GPa}$ respectively.

The change in the spectral position of Raman line at the boundary 4-5 shows that there is a narrow interface layer experiencing strong deformation of expansion. This swelled layer is sandwiched by two adjacent compressed regions in layers 5 and 6 . This formation looks like a long-range compression-expansion wave propagating through the whole layer 5 and disappearing in layer 6 .

It is remarkable that the compressed region of layer 4 does not exhibit strong anomalous birefringence. Large areas of layer 4 adjacent to the boundary 4-5 do not show anomalous birefringence at all. The absence of anomalous birefringence is the manifestation of unchanged symmetry of diamond cubic lattice, which is possible only in the case of hydrostatic stress. The strong anomalous birefringence on the side of layer 5 is an indicator of non-hydrostatic character of the tension in layer 5 .

Similar to the color pattern, the birefringence pattern also shows striations (Fig. 3). In most cases, the birefringence striations follow the direction of color striations. The intensity of anomalous birefringence is the strongest in layers 5 and 6, where the brown/gray color is the most intense. Thus, we conclude that the carbon nanoinclusions and vacancy nanoclusters are an important source of stress observed in CVD diamond.

The birefringence and color patterns do not correlate perfectly. This suggests that carbon and vacancy nanoclusters are not the only cause of internal stress. The other agents could be dislocations and nitrogen. Dislocations can add to the stress substantially without deteriorating color quality [57]. Direct influence of incorporated nitrogen upon internal stress is expected to be negligible because of its low concentration. As shown in $[58,59]$, the nitrogen-related broadening of Raman line in diamond becomes substantial only at concentrations of C-defects over $100 \mathrm{ppm}$. However, an indirect influence of nitrogen, e.g. via the nitrogen-induced twinning, or via the nitrogen-stimulated formation of vacancy clusters, is a possibility.

\subsection{Nitrogen doping}

The efficiency of nitrogen incorporation in our sample, as measured for the total nitrogen concentration with respect to the carbon concentration, is about $10^{-2}$. However, when measured for the optically and paramagnetically active C-defects, it then drops down to $10^{-3}$. The latter value is in accord with most previous reports on nitrogen-doped single crystal CVD diamonds (e.g. [8,60-62]). The difference between the total nitrogen content and the concentration of C-defects is remarkable. Other substitutional nitrogen point defects, NV, NVH, generally make a small fraction of the detected C-defects [8]. The difference between the total nitrogen concentration and C-defect concentration is especially great for low nitrogen concentration in gas. Another remarkable distinction between the total nitrogen concentration and C-defect concentration is that the total concentration weakly depends on the nitrogen content in gas, whereas the C-defect concentration is actually proportional to it. These facts suggest that there are at least two different mechanisms of nitrogen doping of CVD diamond.

The strong non-uniformity of nitrogen doping following the color pattern is likely the result of the terraced character of growth of CVD diamond [12]. The (111)-oriented edges of terraces are more effective in capturing nitrogen than their (100)-oriented flats resulting in the layered incorporation of nitrogen. The differences between the color distribution and the distribution of the total nitrogen concentration suggest that the terraced growth mechanism is not the only explanation 
of the nitrogen non-homogeneity.

It has been discussed in numerous publications that the incorporation of nitrogen in CVD diamond depends on many factors including the nature of the reactive species and their flux to the growth surface, the local stereochemistry, the energetics of the bonding of the reactive species to the surface, and potential etching of bonded species. Experimentally, it has been observed that growth on (111)-oriented planes or local sites incorporates nitrogen as an impurity much more likely than nominal (100) surfaces [63,64]. Since the CVD diamond growth discussed in this work and in the references was performed at temperatures above $800{ }^{\circ} \mathrm{C}$, species reacting with the surface must form relatively strong chemical bonds to the surface to survive long enough to get grown into the bulk. While the growth of single crystal CVD diamonds is usually performed on nominal (100) diamond surfaces, these surfaces are generally not smooth, often displaying macro-steps and island edge contours on the surface [8]. The stereochemistry of these localities and edges is different from a (100) surface looking rather like (111), (113), or even (110) surfaces [64-66]. Additionally, it has been observed by many groups that trace additions of nitrogen ( 0 to $10 \mathrm{ppm}$ ) to the gas reactants can dramatically increase the growth rate and change the surface morphology $[67,68]$. A mechanism of the catalytic role of $\mathrm{N}$ for the growth rate on (111) surfaces has been discussed in [69]. And yet all these proposed models and mechanisms of doping cannot explain the pronounced differences between the total nitrogen concentration and the concentration of substitutional nitrogen point defects as well as the very strong non-homogeneity of nitrogen distribution described above.

We propose an explanation for the above experimental observations assuming two major mechanisms of nitrogen doping for CVD diamond. One is the regular doping with atomic nitrogen species (e.g. CN) from plasma. The other one is the incorporation of carbon nanoclusters containing and/or terminated with nitrogen. The first mechanism is likely responsible for the single nitrogen defects (e.g. C-defects, NV, $\mathrm{NVH}$ ). As for any regular atomic doping during crystal growth, the concentration of dopant in crystal is proportional to the concentration of dopant in growth medium. This is what is observed for the concentration of C-defects (Fig. 2b).

Both doping with single nitrogen species and doping via incorporation by carbon nanoclusters could be responsible for the total concentration of nitrogen. However, since the total nitrogen concentration is much higher than the C-defect concentration, we suggest that the major amount of nitrogen comes into diamond associated with the carbon nanoclusters. The nitrogen in the non-diamond carbon is likely not optically active. The $\mathrm{C}-\mathrm{N}$ atomic bonds holding nitrogen atoms on carbon nanocluster are very strong and nitrogen is not released into diamond lattice to form optically active point defects. Thus, this nitrogen is not detected optically. However, it is readily measured by SIMS. This may explain why the nitrogen concentration measured by SIMS follows intensity of the gray absorption continuum (Fig. 8b). In both cases, the intensity of SIMS signal and the strength of the gray absorption continuum depend on the concentration of carbon nanoclusters.

The area probed by SIMS instrument is a few micrometers in size which is comparable with the assumed average distance between the carbon nanoclusters. This explains why the nitrogen concentration measured by SIMS is so fluctuating. The SIMS signal simply follows the random distribution of carbon nanoclusters.

The nitrogen doping via incorporation of carbon nanoclusters can also explain the weak dependence of the total nitrogen concentration in diamond on the total concentration in gas. If the carbon nanoclusters are more reactive to nitrogen species either in the plasma or on the surface, then the surface of nanoclusters becomes rapidly saturated with nitrogen and the amount of nitrogen entering diamond becomes weakly dependent on the nitrogen concentration in gas.

We suggest that the carbon nanocluster mechanism of doping can work for other impurities too, for instance, for hydrogen and oxygen.
There are experimental observations suggesting this similarity for nitrogen and hydrogen doping. For instance, the efficiency of doping of CVD diamond with hydrogen decreases with the increase in the gas flow rate in the same way as for nitrogen [46]. Hydrogen is also known as a specie effectively terminating carbon nanoparticles. This termination is stable enough and carbon nanoclusters do not release hydrogen upon being grown-in in diamond. Thus, the measurements of the total hydrogen concentration in CVD diamond by direct methods (SIMS, NMR, ion scattering), which are not sensitive to the atomic configuration of impurity, always yield much higher values (up to a few tenths of percent in polycrystalline diamond) than the spectroscopic methods (optical absorption, EPR on single crystal diamond), which never reveal hydrogen concentration over a few tens of ppm (e.g. [70]).

\section{Conclusion}

A $4.3 \mathrm{~mm}$ thick block of single crystal CVD diamond was grown in 6 layers with increasing nitrogen concentration in the gas reactants. The sample was studied using Raman, SIMS, UV-Vis absorption, and birefringence imaging. The total nitrogen concentration as measured by SIMS was highly variable within each growth layer but generally increased with the nitrogen added to the reactants. The brown/gray color of the diamond was also highly variable and generally increasing with the gas phase nitrogen concentration. This color is attributed to multiple absorption components: a gray absorption continuum, a brown absorption continuum, and absorption by defects containing substitutional nitrogen atoms. It was found that the optically active nitrogen present in form of point lattice defects was only a small fraction of the total nitrogen in the diamond.

It is suggested that the brown color is due to vacancy clusters similar to those in brown natural diamonds but smaller in size. While absorption on the vacancy clusters is believed to be the main reason of brown color, Mie scattering could be also a contributor. The gray color is assumed to be due to pi-bonded $\mathrm{sp}^{2}$ carbon nanoclusters grown-in into diamond. A simulation of Mie light scattering by spherical graphitic nanoparticles of a size of a few tens of nanometers with concentration in the range of $10^{11} \mathrm{~cm}^{-3}$ supports this interpretation. These carbon nanoclusters could form in the plasma or on the growing diamond surface. Indirect evidence seems to support the formation of solid carbon nanoparticles in the plasma.

It is also suggested that the carbon nanoclusters may contain a significant nitrogen or hydrogen component (e.g. in form of surface termination). The incorporation of these terminated carbon nanoclusters may explain the high level of total bulk impurity concentration of impurities detected by SIMS.

It is shown that the brown/gray color and the internal stress in CVD diamond are related effects. The striation pattern of brown/gray color follows the pattern of anomalous birefringence. This similarity suggests that the higher concentration of vacancy clusters and nondiamond carbon inclusions the higher internal stress of crystal lattice. The terraced growth on nominally (100) surfaces is a likely explanation of the formation of striations. The terrace edges oriented along (111) planes are assumed to be the sites of effective trapping of nitrogen and generation of vacancies.

A practically important conclusion is made that the high perfection of seed surface at microscale is not a required condition for the growth of low-stressed CVD diamond single crystal. Crystallographic order at macroscale is much more important. A low-stressed, low-inclusion diamond can be grown even on single crystal surface with high concentration of macro inclusions. This conclusion is in accord with the well-known practice in CVD diamond industry when gem quality CVD diamonds are grown on relatively rough laser-cut seeds without prior polishing. 


\section{Prime novelty and highlights}

1. A model is presented for doping of CVD diamond with nitrogen and other impurities via incorporation of impurity-terminated carbon nanoclusters formed in the plasma, or on the growing surface.

2. Two major components of UV-Vis absorption spectrum of as-grown nitrogen-doped CVD diamond are a brown continuum and a gray continuum. The brown absorption continuum is attributed to small vacancy clusters, while the gray absorption continuum is attributed to carbon nanoclusters.

3. Mie light scattering on carbon nanoclusters and vacancy clusters may be a significant contributor to gray and brown color of CVD diamonds.

4. It is suggested that the growth on terrace edges and micro-steps formed on the surface of growing CVD diamond contributes to its brown color, stress and nitrogen doping.

\section{CRediT authorship contribution statement}

A.M. Zaitsev: Conceptualization, Investigation. N.M. Kazuchits: Investigation. V.N. Kazuchits: Investigation. K.S. Moe: Investigation. M.S. Rusetsky: Investigation, Formal analysis. O.V. Korolik: Investigation. Kouki Kitajima: Investigation, Formal analysis. J.E. Butler: Investigation, Formal analysis. W. Wang: Supervision.

\section{Declaration of competing interest}

The authors confirm that they have no conflicts of interests.

\section{Acknowledgements}

The authors are grateful to Adrian Chan for preparation of samples, Dr. Tingting Gu for help with SIMS measurements and Prof. John Valley for discussion of the SIMS results.

\section{References}

[1] D.G. Goodwin, J.E. Butler, M.A. Prelas, G. Popovici, L.K. Biglow (Eds.), Handbook of Industrial Diamonds and Diamond Films, Marcel Dekker, Inc, NY, 1997, pp. $527-581$.

[2] A.M. Zaitsev, Optical Properties of Diamond: A Data Handbook, Springer-Verlag, Berlin, Heidelberg, 2001, p. 502.

[3] A. Tallaire, A.T. Collins, D. Charles, J. Achard, R. Sussmann, A. Gicquel, M.E. Newton, A.M. Edmonds, R.J. Cruddace, Characterization of high-quality thick single-crystal diamond grown by CVD with a low nitrogen addition, Diamond \& Related Materials 15 (2006) 1700-1707.

[4] C. Glover, M.E. Newton, P.M. Martineau, D.J. Twitchen, J.M. Baker, Hydrogen incorporation in diamond: the nitrogen-hydrogen-vacancy complex, Phys. Rev. Lett. 90 (2003) 185507.

[5] R.U.A. Khan, B.L. Cann, P.M. Martineau, J. Samartseva, J.J.P. Freeth, S.J. Sibley, C.B. Hartland, M.E. Newton, H.K. Dhillon, D.J. Twitchen, Colour-causing defects and their related optoelectronic transitions in single crystal CVD diamond, J. Phys. Condens. Matter 25 (2013) 275801.

[6] K. Iakoubovskii, G.J. Adriaenssens, Optical detection of defect centers in CVD diamond, Diam. Relat. Mater. 9 (2000) 1349-1356.

[7] E. Rohrer, C.F.O. Graeff, R. Jansen, C.E. Nebel, M. Stutzmann, H. Guettler, R. Zachai, Nitrogen-related dopant and defect states in CVD diamond, Phys. Rev. B 54 (1996) 7874-7880.

[8] A. Tallaire, A.T. Collins, D. Charles, J. Achard, R. Sussmann, A. Gicquel, M.E. Newton, A.M. Edmonds, R.J. Cruddace, Characterization of high-quality thick single-crystal diamond grown by CVD with a low nitrogen addition, Diam. Relat. Mater. 5 (2006) 1700-1707.

[9] W. Wang, M.S. Hall, K.S. Moe, J. Tower, T.M. Moses, Latest-generation CVD-grown synthetic diamonds from Apollo Diamond Inc. Gems and Gemology 43 (4) (2007) 294-312.

[10] A.M. Zaitsev, W. Wang, K.S. Moe, P. Johnson, Spectroscopic studies of yellow nitrogen-doped CVD diamonds, Diam. Relat. Mater. 68 (2016) 51-61.

[11] A. Ishida, K. Kitajima, K.H. Williford, M.L. Tuite, T. Kakegawa, J.W. Valley, Simultaneous in situ analysis of carbon and nitrogen isotope ratios in organic matter by secondary ion mass spectrometry, Geostand. Geoanal. Res. 42 (2018) 189-203.

[12] P.M. Martineau, S.C. Lawson, A.J. Taylor, S.J. Quinn, D.J.F. Evans, M.J. Crowder, Identification of synthetic diamond grown using chemical vapor deposition (CVD), Gems \& Gemology 40 (1) (2004) 2-25.

[13] I.A. Dobrinets, V.G. Vins, A.M. Zaitsev, HPHT Treated Diamonds, Springer-Verlag,
Berlin Heidelberg, 2013, p. 257.

[14] W. Wang, T. Moses, R. Linares, J.E. Shigley, M. Hall, J.E. Butler, Gem-quality synthetic diamonds grown by the chemical vapor deposition method, Gems and Gemology 39 (4) (2003) 268-283.

[15] W. Wang, P. Doering, J. Tower, R. Lu, S. Eaton-Magaña, P. Johnson, E. Emerson, T.M. Moses, Strongly colored pink CVD lab-grown diamonds, Gems \& Gemology 46 (1) (2010) 4-17.

[16] W. Wang, A. Tallaire, M.S. Hall, T.M. Moses, J. Achard, R.S. Sussmann, A. Gicquel, Experimental CVD synthetic diamonds from LIMHP-CNRS, France, Gems \& Gemology 41 (3) (2005) 2-12.

[17] A. Tallaire, J. Achard, A. Boussadi, O. Brinza, A. Gicquel, I.N. Kupriyanov, Y.N. Palyanov, G. Sakr, J. Barjon, High quality thick CVD diamond films homoepitaxially grown on (111)-oriented substrates, Diam. Relat. Mater. 41 (2014) 34-40.

[18] O. Brinza, J. Achard, F. Silva, X. Bonnin, P. Barroy, K.D. Corte, A. Gicquel, Dependence of CVD diamond growth rate on substrate orientation as a function of process parameters in the high microwave power density regime, Phys. Status Solidi (a) 205 (2008) $2114-2120$.

[19] I. Kiflawi, H. Kanda, S.C. Lawson, The effect of the growth rate on the concentration of nitrogen and transition metal impurities in HPHT synthetic diamonds, Diam. Relat. Mater. 11 (2002) 204-211.

[20] Y. Mokuno, A. Chayahara, H. Yamada, N. Tsubouchi, Large single crystal diamond plates produced by microwave plasma CVD, Mater. Sci. Forum 615-617 (2009) 991-994.

[21] F. De Weerdt, J. Van Royen, Defects in coloured natural diamonds, Diam. Relat. Mater. 10 (2001) 474-479.

[22] D. Fisher, S.J. Sibley, C.J. Kelly, Brown colour in natural diamond and interaction between the brown related and other colour-inducing defects, J. Phys.: Condense Matter. 21 (2009) 364213.

[23] L.S. Hounsome, R. Jones, P.M. Martineau, D. Fisher, M.J. Shaw, P.R. Briddon, S. Öberg, Role of extended defects in brown colouration of diamond, Phys. Status Solidi C 4 (2007) 2950-2957.

[24] R. Jones, Dislocations, vacancies and the brown colour of CVD and natural diamond, Diamond \& Related Materials 18 (2009) 820-826.

[25] U. Bangert, R. Barnes, L.S. Hounsome, R. Jones, A.T. Blumenau, P.R. Briddon, M.J. Shaw, S. Öberg, Phila. Mag. 86 (2006) 4757.

[26] C.F. Bohren, D.R. Huffmann, Absorption and Scattering of Light by Small Particles, Wiley-Interscience, New York, 2010, p. 544.

[27] S. Prahl, Mie Scattering Calculator, https://omlc.org/calc/mie_calc.html.

[28] K. Meykens, M. Nesladek, C. Quaeyhaegens, L.M. Stals, M. Vanecek, J. Rosa, G. Janssen, J.J. Schermer, Optical absorption and cathodoluminescence in homoepitaxially grown CVD diamond films, Diam. Relat. Mater. 5 (1996) 958-963.

[29] Th. Hainschwang, E. Fritsch, F. Notari, B. Rondeau, A. Katrusha, The origin of color in natural C center bearing diamonds, Diamond \& Related Materials 39 (2013) $27-40$.

[30] R.C. Burns, V. Cvetkovic, C.N. Dodge, D.J.F. Evans, M.-L.T. Rooney, P.M. Spear, C.M. Welbourn, Growth-sector dependence of optical features in large synthetic diamonds, J. Cryst. Growth 104 (1990) 257-279.

[31] R.U. Khan, P.M. Martineau, B.L. Cann, M.E. Newton, D.J. Twitchen, Charge transfer effects, thermo and photochromism in single crystal CVD synthetic diamond, J Phys Condens. Matter. 21 (2009) 364214.

[32] A.T. Collins, Colour centers in diamond, J. of Gemmology 18 (1982) 37-75.

[33] M.N. Polyanskiy, Refractive index database, https://refractiveindex.info , Accessed date: 29 November 2019.

[34] C.H. van der Bogert, C. Smith, T. Hainschwang, S.F. McClure, Gray-to-blue-to-violet hydrogen-rich diamonds from the Argyle Mine, Australia, Gems and Gemology 45 (1) (2009) 20-37.

[35] K. Iakoubovskii, G.J. Adriaenssens, Optical characterization of natural Argyle diamonds, Diam. Relat. Mater. 11 (2002) 125-131.

[36] K.V. Smit, S.B. Shirey, R.A. Stern, A. Steele, W. Wang, Diamond growth from C-H-N$\mathrm{O}$ recycled fluids in the Zimbabwe lithosphere: evidence from CH4 micro-inclusions and $813 \mathrm{C}-815 \mathrm{~N}-\mathrm{N}$ content in Marange mixed-habit diamonds, Lithos 265 (2016) 68-81.

[37] V.G. Vins, O.V. Kononov, A model of HPHT color enhancement mechanism in natural gray diamonds, Diam. Relat. Mater. 12 (2003) 542-545.

[38] T. Stachel, R.W. Luth, Diamond formation - where, when and how? Lithos 220 (223) (2015) 200-220.

[39] S. Prawer, K.W. Nugent, D.N. Jamieson, The Raman spectrum of amorphous diamond, Diam. Relat. Mater. 7 (1998) 106-110.

[40] L. Pereira, E. Pereira, A. Cremades, J. Piqueras, J. Jiménez, J.M. Bielza, Characterization of different habits in torch-flame-grown diamond and diamondlike films, Diam. Relat. Mater. 8 (1999) 1333-1341.

[41] P. Bou, L. Vandenbulcke, Raman investigations on diamond films and crystals deposited by plasma-assisted CVD, J. Electrochem. Soc. 138 (1991) 2991-3000.

[42] Y. Gogotsi, K.G. Nickel, Pressure-induced phase transformations in diamond, J. Appl. Phys. 84 (1998) 1299-1304.

[43] P.K. Bachmann, D.U. Wiechert, Optical characterization of diamond, Diam. Relat. Mater. 1 (1992) 422-443.

[44] L.C. Nistor, J. Van Landuyt, V.G. Ralchenko, E.D. Obraztsova, A.A. Smolin, Nanocrystalline diamond films: transmission electron microscopy and Raman spectroscopy characterization, Diam. Relat. Mater. 6 (1997) 159-168.

[45] K. Okada, S. Komatsu, T. Ishigaki, S. Matsumoto, Y. Moriyoshi, Evaluation of the surface structure of diamond films prepared in a combustion flame by surface-enhanced Raman scattering, Appl. Phys. Lett. 60 (1992) 959-961.

[46] S.V. Nistor, M. Stefan, V. Ralchenko, A. Khomich, D. Schoemaker, Nitrogen and hydrogen in thick diamond films grown by microwave plasma enhanced chemical 
vapor deposition at variable $\mathrm{H}_{2}$ flow rates, J. Appl. Phys. 87 (2000) 8741-8746.

[47] S.V. Nistor, J. Van Landuyt, V. Ralchenko, Structural aspects of CVD diamond wafers grown at different hydrogen flow rates, Phys. Status Solidi A 174 (1999) 5-9.

[48] M.N.R. Ashfold, E.J.D. Mahoney, S. Mushtaq, B.S. Truscott, Y. Mankelevich, What [plasma used for growing] diamond can shine like flame? Chem. Commun. 53 (2017) 10482-10495.

[49] R. Vander Wal, A. Sengupta, E. Musselman, G. Skoptsov, Microwave-driven plasmamediated methane cracking: product carbon characterization, C-Journal of Carbon Research 4 (61) (2018) 16.

[50] M. Gautier, V. Rohani, L. Fulcheri, J.P. Trelles, Influence of temperature and pressure on carbon black size distribution during allothermal cracking of methane, Aerosol Sci. Technol. 50 (2016) 26-40.

[51] J.W. Ager III, M.D. Drory, Quantitative measurement of residual biaxial stress by Raman spectroscopy in diamond grown on a Ti alloy by chemical vapor deposition, Phys. Rev. B 48 (1993) 2601-2607.

[52] F. Ahmed, K. Durst, S.M. Rosiwal, M. Göken, J. Fandrey, J. Schaufler, In-situ tensile testing of crystalline diamond coatings using Raman spectroscopy, Surf. Coat. Technol. 204 (2009) 1022-1025.

[53] E.J. Di Liscia, F. Álvarez, E. Burgos, E.B. Halac, H. Huck, M. Reinoso, Stress analysis on single-crystal diamonds by Raman spectroscopy 3D mapping, Mater. Sci. Appl. 4 (2013) 191-197.

[54] J.M. Boteler, Y.M. Gupta, Shok-induced splitting of the triply degenerate Raman line in diamond, Phys. Rev. Lett. 71 (1993) 3497-3500.

[55] G. Davies, Current problems in diamond: towards a quantitative understanding, Physica B 273/274 (1999) 15-23.

[56] M.H. Grimsditch, E. Anastassakis, M. Cardona, Effect of uniaxial stress on the zonecenter optical phonon of diamond, Phys. Rev. B 18 (1978) 901-904.

[57] P.M. Martineau, M.P. Gaukroger, K.B. Guy, S.C. Lawson, D.J. Twitchen, I. Friel, J.O. Hansen, G.C. Summerton, T.P.G. Addison, R. Burns, High crystalline quality single crystal chemical vapour deposition diamond, J. Phys. Condens. Matter 21 (2009) 364205.

[58] N.V. Surovtsev, I.N. Kupriyanov, V.K. Malinovsky, V.A. Gusev, Y. Pal'yanov, Effect of nitrogen impurities on the Raman line width in diamonds, J. Phys. Condens. Matter 11 (1999) 4767-4774.
[59] M. Muehle, M.K. Yaran, M.F. Becker, T. Schuelke, J. Asmussen, Y. Gu, D.K. Reinhard, T.A. Grotjohn, Quality and internal stress of single crystalline diamond synthesized by microwave plasma assisted chemical vapor deposition, Poster. available online at http://mipse.umich.edu/files/MIPSE_GS2012_Muehle2.pdf.

[60] A. Bergmaier, G. Dollinger, T. Faestermann, C.M. Frey, M. Ferguson, H. Giittler, G. Schulz, H. Willerscheid, Detection of nitrogen in CVD diamond, Diam. Relat. Mater. 5 (1996) 995-997.

[61] M.A. Lobaev, A.M. Gorbachev, S.A. Bogdanov, A.L. Vikharev, D.B. Radishev, V.A. Isaev, V.V. Chernov, M.N. Drozdov, Influence of CVD diamond growth conditions on nitrogen incorporation, Diamond \& Related Materials 72 (2017) 1-6.

[62] R. Samlenski, C. Haug, R. Brenn, C. Wild, R. Lecher, P. Koidl, Characterization and lattice location of nitrogen and boron in homoepitaxial CVD diamond, Diam. Relat. Mater. 5 (1996) 947-951.

[63] J.E. Butler, A. Cheesman, M.N.R. Ashfold, Recent progress in the understanding of CVD growth of diamond, in: R.S. Sussmann (Ed.), CVD Diamond for Electronic Devices and Sensors, John Wiley \& Sons, Chichester UK, 2009, pp. 103-124.

[64] J.E. Butler, Y.A. Mankelevich, A. Cheesman, J. Ma, M.N.R. Ashfold, Understanding the chemical vapor deposition of diamond: recent progress, J. Phys. Condens. Matter 21 (2009) 364201

[65] C. Wild, N. Herres, P. Koidl, Texture formation in polycrystalline diamond films, J Appl. Phys. 68 (1990) 973-978.

[66] C. Wild, R. Kohl, N. Herres, W. Muller-Sebert, P. Koidl, Oriented CVD diamond films - twin formation, structure and morpholooy, Diam. Relat. Mater. 3 (1994) 373-381.

[67] A.F. Sartori, M. Schreck, Mutual interaction of N, B, and O during heteroepitaxial diamond growth: triggering the nitrogen induced growth acceleration, Phys. Status Solidi A 211 (2014) 2290-2295.

[68] J. Achard, F. Silva, O. Brinza, A. Tallaire, A. Gicquel, Coupled effect of nitrogen addition and surface temperature on the morphology and the kinetics of thick CVD diamond single crystals, Diam. Relat. Mater. 16 (2007) 685-689.

[69] J.E. Butler, I. Oleynik, A mechanism for crystal twinning in the growth of diamond by chemical vapour deposition, Philos. Trans. R. Soc. A Math. Phys. Eng. Sci. 366 (2008) 295-310.

[70] X. Zhou, G.D. Watkins, K.M. McNamara, R.P. Messmer, S. Chawla, Hydrogen-related defects in polycrystalline CVD diamond, Phys. Rev. B 54 (1996) 7881-7890. 